\title{
Role of Glial Cell Line-Derived Neurotrophic Factor (GDNF)-Neural Cell Adhesion Molecule (NCAM) Interactions in Induction of Neurite Outgrowth and Identification of a Binding Site for NCAM in the Heel Region of GDNF
}

\author{
Janne Nielsen, Kamil Gotfryd, Shizhong Li, Nikolaj Kulahin, Vladislav Soroka, Kim K. Rasmussen, Elisabeth Bock, \\ and Vladimir Berezin \\ Protein Laboratory, Department of Neuroscience and Pharmacology, Faculty of Health Sciences, University of Copenhagen N, DK-2200 Copenhagen, \\ Denmark
}

The formation of appropriate neuronal circuits is an essential part of nervous system development and relies heavily on the outgrowth of axons and dendrites and their guidance to their respective targets. This process is governed by a large array of molecules, including glial cell line-derived neurotrophic factor (GDNF) and the neural cell adhesion molecule (NCAM), the interaction of which induce neurite outgrowth. In the present study the requirements for NCAM-mediated GDNF-induced neurite outgrowth were investigated in cultures of hippocampal neurons, which do not express Ret. We demonstrate that NCAM-mediated GDNF-induced signaling leading to neurite outgrowth is more complex than previously reported. It not only involves NCAM-140 and the Src family kinase Fyn but also uses NCAM-180 and the fibroblast growth factor receptor. We find that induction of neurite outgrowth by GDNF via NCAM or by transhomophilic NCAM interactions are not mutually exclusive. However, whereas NCAM-induced neurite outgrowth primarily is mediated by NCAM-180, we demonstrate that GDNF-induced neurite outgrowth involves both NCAM-140 and NCAM-180. We also find that GDNF-induced neurite outgrowth via NCAM differs from NCAM-induced neurite outgrowth by being independent of NCAM polysialylation. Additionally, we investigated the structural basis for GDNF-NCAM interactions and find that NCAM Ig3 is necessary for GDNF binding. Furthermore, we identify within the heel region of GDNF a binding site for NCAM and demonstrate that a peptide encompassing this sequence mimics the effects of GDNF with regard to NCAM binding, activation of intracellular signaling, and induction of neurite outgrowth.

\section{Introduction}

Glial cell line-derived neurotrophic factor (GDNF) promotes the survival and affects the proliferation, migration, and differentiation of a number of neuronal populations within the central and peripheral nervous systems (for review, see Airaksinen and Saarma, 2002; Enomoto, 2005; Paratcha and Ledda, 2008). GDNF, together with three related molecules, neurturin, artemin, and persephin, constitutes the GDNF family ligands (GFLs) (Airaksinen et al., 1999). All GFLs signal through a receptor complex consisting of a ligand binding GDNF family receptor $\alpha$ $(\mathrm{GFR} \alpha)$ and a signal transducing molecule, the receptor tyrosine kinase Ret. Four different GFR $\alpha$ subtypes (1-4) exist, and each

Received July 8, 2009; accepted July 29, 2009.

This work was supported by "Snedkermester Sophus Jacobsen og hustru Astrid Jacobsens Fond," “Det Lægevidenskabelige Fakultets Fond for videnskabeligt ansatte kandidater og studerende ved Københavns Universitet," ENKAM Pharmaceuticals A/S, the Graduate School of Neuroscience of University of Copenhagen, and the European Union's Sixth Framework Programme PROMEMORIA (LSHM-CT-2005-512012). We thank Stine M. Hansen, Lene Køhler, and Peter S. Walmod for technical support and fruitful discussions.

Correspondence should be addressed to Janne Nielsen, Protein Laboratory, Department of Neuroscience and Pharmacology, Faculty of Health Sciences, University of Copenhagen, Panum Institute, Building 24.2, Blegdamsvej 3C, DK-2200 Copenhagen N, Denmark. E-mail: jannen@sund.ku.dk.

DOI:10.1523/JNEUROSCI.3239-09.2009

Copyright $\odot 2009$ Society for Neuroscience $\quad 0270-6474 / 09 / 2911360-17 \$ 15.00 / 0$ bind their preferred GFL, thereby conferring ligand specificity to the receptor complex, although some cross talk can occur (for review, see Airaksinen et al., 1999).

GFR $\alpha$ is more widely expressed than Ret (Nosrat et al., 1997; Trupp et al., 1997; Yu et al., 1998), and GDNF has been found to signal independently of Ret (Poteryaev et al., 1999; Trupp et al., 1999; Pezeshki et al., 2001). This suggests the existence of additional GDNF signaling receptors, and the neural cell adhesion molecule (NCAM) has been identified as an alternative receptor for GDNF (Paratcha et al., 2003).

NCAM plays an important role in nervous system morphogenesis and affects neural regeneration and plasticity, including learning and memory (for review, see Maness and Schachner, 2007; Conboy et al., 2008; Hartz and Rønn, 2008). The NCAM ectodomain consists of five N-terminal Ig-like modules (Ig1-5) followed by two membrane-proximal fibronectin type III homology modules (FN3-1 and FN3-2). NCAM exists in three major isoforms with identical ectodomains: NCAM-140 and NCAM180 are transmembrane molecules, and NCAM-120 is linked to the plasma membrane by a glycosylphosphatidyl inositol anchor (Cunningham et al., 1987). NCAM is subject to several posttranslational modifications, most prominently the addition of polysi- 
alic acid (PSA), that have profound effects on NCAM function (for review, see Rutishauser, 2008). NCAM promotes cell adhesion through homophilic interactions (for review, see Soroka et al., 2008). Additionally, NCAM triggers intracellular signal transduction and modulation of cytoskeletal organization by interacting with numerous heterophilic partners (for review, see Büttner and Horstkorte, 2008; Ditlevsen et al., 2008; Nielsen et al., 2008). The most studied signaling pathways are the signaling through the Src family kinase Fyn (Beggs et al., 1997), and the fibroblast growth factor receptor (FGFR) (Kiselyov et al., 2003), both of which have been found to be essential for NCAMinduced neurite outgrowth (Kolkova et al., 2000a; Niethammer et al., 2002). FGFR interacts directly with NCAM (Kiselyov et al., 2003), whereas Fyn is activated by NCAM through receptor protein tyrosine phosphatase $\alpha$ (Bodrikov et al., 2005).

Previous studies on GDNF-NCAM interactions demonstrated that NCAM-mediated GDNF signaling leads to neurite outgrowth from embryonic hippocampal and cortical neurons (Paratcha et al., 2003). Here, we report that both NCAM-140 and NCAM-180 mediated GDNF-induced neurite outgrowth from hippocampal neurons and that downstream signaling involved both Fyn and FGFR, whereas NCAM polysialylation did not appear to be required for the effect of GDNF on neurite outgrowth.

Using surface plasmon resonance (SPR) analysis, we found, in accordance with Sjöstrand et al. (2007), that NCAM Ig3 is necessary for GDNF binding. We identified a motif in the primary sequence of GDNF that is involved in binding to NCAM and demonstrate that a peptide, termed Gliafin, encompassing this motif, mimics GDNF with regard to binding, signaling, and effects on neurite outgrowth.

The present data substantiate the role of NCAM as a GDNF receptor and indicate the importance of GDNF-NCAM interactions in nervous system development. Furthermore, the data underline the role of NCAM as a molecule integrating long-range signals provided by growth factors and short-range signals provided by adhesive interactions. Such integration may not only be important during development but might also play an important role in neural plasticity, learning and memory, and maintenance of the nervous system, which have all been shown to be affected by GDNF and NCAM.

\section{Materials and Methods}

\section{Materials}

The Fyn kinase inhibitor [4-amino-5-4-chlorophenyl]-7[t-butyl]pyrazolo[3,4d]pyrimidine (PP2), the FGFR inhibitor 3-[3-(2-carboxyythyl)-4methylpyrrol-2-methylidenyl]-2-indolinone (SU5402), and the phosphatase inhibitor mixture set III were purchased from Calbiochem. The Complete EDTA-free protease inhibitor mixture was purchased from Roche. B27 supplement, fetal calf serum (FCS), horse serum, fungizone, GlutaMAX, HEPES, Neurobasal medium, geneticin, and penicillin/streptomycin were obtained from Invitrogen. Trypsin, DNase 1, soybean trypsin inhibitor, bovine serum albumin (BSA), Coomassie brilliant blue R-250, NP-40, and colominic acid were obtained from SigmaAldrich. Human recombinant GFR $\alpha 1 /$ Fc chimera, human recombinant GDNF (for SPR analysis), and human recombinant FGF2 were purchased from R\&D Systems. For cellular experiments, human recombinant GDNF was purchased from PeproTech. The sequences of the peptides Gliafin (76-ETMYDKILKNLSRSR-90), Finger 1 peptide (48LNVTDLGLGYETKEE-62), and Finger 2 peptide (110-DLSFLDDSLVY120) were derived from rat GDNF [Protein Data Bank (PDB) 1AGQ], and the peptides were produced as tetrameric dendrimers coupled to a lysine backbone (Schafer-N). A scrambled version (KRDLTISNSMEKYRL) and truncated forms (MYDKILKNLSRSR, DKILKNLSRSR, ILKNLSRSR, ETMYDKILKNLSR, ETMYDKILKNL, ETMYDKILK) of the Gliafin peptide were synthesized in the same way.

\section{Recombinant proteins}

The cDNA fragments encoding NCAM Ig3-5 without the variable alternatively spliced exon (VASE) (residues 213-511; Swiss-Prot P13596) and NCAM Ig1-5 with and without the VASE (residues 20-511; Swiss-Prot P13596), all with a C-terminal His tag, were synthesized by PCR using rat NCAM-120 (without the alternatively spliced VASE) or NCAM-140 (with the alternative spliced VASE) cDNA as a template. The amplified cDNA fragments were subcloned into the SmaI/XhoI site (NCAM Ig3-5 construct) of pMT/BiP/V5-His A plasmid (Invitrogen) or into the KpnI/ XhoI site (NCAM Ig1-5 constructs) of pMT/V5-His A plasmid (Invitrogen). Recombinant proteins were expressed in Drosophila S2 cells and purified as previously described (Kulahin et al., 2004).

Recombinant rat NCAM Ig1-3 (residues 20-308; Swiss-Prot P13596) protein was produced as previously described (Soroka et al., 2003).

\section{Cell cultures}

Primary cultures of hippocampal neurons. Hippocampal neurons were obtained from embryonic day 19 Wistar rat embryos (Charles River Laboratories) as previously described (Maar et al., 1997). Briefly, embryos were decapitated, and the brains were removed. Hippocampi were dissected and cleared from membranes and blood vessels in ice-cold modified Krebs'-Ringer's buffer. Tissue dissociation was performed by chopping and trypsinization, and trypsinization was stopped with Krebs'Ringer's buffer containing soybean trypsin inhibitor and DNase I. Undissociated tissue was pelleted by centrifugation, and neurons were resuspended in Krebs'-Ringer's buffer with $\mathrm{Ca}^{2+}$ and $\mathrm{Mg}^{2+}$. Neurons were then pelleted and resuspended in Neurobasal medium with supplements [2\% (v/v) B27, 0.4\% (w/v) BSA, 2 mм GlutaMAX, 20 mм HEPES, $100 \mathrm{U} / \mathrm{ml}$ penicillin, and $100 \mu \mathrm{g} / \mathrm{ml}$ streptomycin].

Cell lines. An NCAM-expressing cell line (LBN110; hereafter denoted LBN) has been generated from the mouse fibroblastoid cell line L929 by stable transfection with a eukaryotic expression vector encoding human NCAM-140 (without the exons VASE, a, b, c, and AAG). The corresponding control cell line (LVN212; hereafter denoted LVN) was generated by stable transfection of L929 cells with the empty vector (Kasper et al., 1996). LVN and LBN cells were propagated in DMEM supplemented with $10 \%$ (v/v) FCS, $2 \mathrm{~mm}$ GlutaMAX, $100 \mathrm{U} / \mathrm{ml}$ penicillin, $100 \mu \mathrm{g} / \mathrm{ml}$ streptomycin, $2.5 \mu \mathrm{g} / \mathrm{ml}$ fungizone, and $567 \mu \mathrm{g} / \mathrm{ml}$ geneticin.

T-REx-293 cells (Invitrogen) were stably transfected with human FGFR1 containing a C-terminal Strep II tag as previously described (Kiselyov et al., 2003) and propagated in DMEM with 10\% (v/v) FCS, 2 mM GlutaMAX, $200 \mu \mathrm{g} / \mathrm{ml}$ hygromycin, $100 \mathrm{U} / \mathrm{ml}$ penicillin, and 100 $\mu \mathrm{g} / \mathrm{ml}$ streptomycin.

\section{Transfection of hippocampal neurons}

Hippocampal neurons $\left(3-5 \times 10^{6}\right)$ were transfected with $3 \mu \mathrm{g}$ of DNA by electroporation using a nucleofector device and a Rat Neuron Nucleofector kit (Amaxa). A vector encoding short hairpin RNA (shRNA) for NCAM (Hansen et al., 2007) was used to knock down the expression of NCAM. A vector encoding shRNA not targeting any known expressed rat sequence (Hansen et al., 2007) was used as a control. For uncoupling of signaling through NCAM-180 and NCAM-140, respectively, expression vectors encoding the cytoplasmic domain of either NCAM-180 or NCAM-140 (Kolkova et al., 2000b) were used, and a matching empty vector served as a control. The expression of the cytoplasmic domains by this method has been confirmed previously (Kolkova et al., 2000b; Prag et al., 2002; Büttner et al., 2004). An expression vector encoding a dominant-negative version of Fyn (dnFyn) (Addgene plasmid 16033) (Mariotti et al., 2001) was used to inhibit Fyn, and a matching empty vector was used as a control. An expression vector encoding a kinasedeficient dominant-negative version of FGFR (dnFGFR) (Saffell et al., 1997) was used to inhibit FGFR, and a matching empty vector was applied as a control. To identify transfected cells, all cells were cotransfected with $0.5 \mu \mathrm{g}$ of an expression vector (p-EGFP-N1; Clontech) encoding the enhanced variant of the Aequorea victoria green fluorescent protein (GFP). Transfected neurons were incubated in neurobasal medium with supplements and $5 \%(\mathrm{v} / \mathrm{v})$ FCS for $2 \mathrm{~h}$ at $37^{\circ} \mathrm{C}$ before being used for experiments. 


\section{Neurite outgrowth}

For monoculture assays, primary hippocampal neurons in Neurobasal medium with supplements were seeded in Permanox Lab-Tek tissue culture chamber slides (Nalge Nunc International) at a density of $8 \times 10^{4}$ cells $/ \mathrm{cm}^{2}$. For coculture assays, hippocampal neurons were seeded in Neurobasal medium with supplements on top of a confluent layer of LVN or LBN cells. Nontransfected neurons were seeded at a density of $5.6 \times 10^{4}$ cells $/ \mathrm{cm}^{2}$; neurons transfected with vectors encoding cytoplasmic domains of NCAM-180 or NCAM-140 or dnFGFR were seeded at $1.1 \times 10^{5}$ cells $/ \mathrm{cm}^{2}$; neurons transfected with plasmids encoding dnFyn or NCAM shRNA were seeded at $3.3 \times 10^{5} \mathrm{cells} / \mathrm{cm}^{2}$. For enzymatic removal of PSA from NCAM, 60 nm endoneuraminidase-N (EndoN) (a kind gift from Prof. Dr. Rita Gerardy-Schahn, Hannover Medical School, Hannover, Germany) was included in the medium from the onset of the experiments.

Unless otherwise stated, GDNF (PeproTech) or peptides were added to cultures immediately after seeding. For experiments involving pharmacological inhibitors, these were added to the cultures $10 \mathrm{~min}$ before GDNF or peptide. Neurons transfected with vectors encoding cytoplasmic domains of NCAM-180 or NCAM-140 or dnFGFR were grown for $6 \mathrm{~h}$ before GDNF or Gliafin was added.

\section{Fluorescence staining and image processing}

Neurons were grown for $24 \mathrm{~h}$ at $37^{\circ} \mathrm{C}$ in $5 \% \mathrm{CO}_{2}$ before being fixed for 20 $\mathrm{min}$ at room temperature in fixation buffer $[3.7 \%(\mathrm{v} / \mathrm{v})$ formaldehyde, $1 \%$ (v/v) methanol, $0.4 \mathrm{~mm} \mathrm{CaCl}_{2}$, and $0.05 \mathrm{~m}$ sucrose in PBS]. Depending on the experiment, cells were incubated overnight at $4^{\circ} \mathrm{C}$ with either rabbit anti-growth-associated protein (GAP)-43 antibody (Millipore Bioscience Research Reagents; nontransfected neurons) or mouse antiGFP antibody (Roche; transfected neurons), and visualized with Alexaconjugated goat anti-rabbit or goat anti-mouse antibodies (Invitrogen), and mounted with anti-fade mounting medium (Dako). Alternatively, cells were stained with Coomassie $[0.5 \%(\mathrm{w} / \mathrm{v})$ Coomassie brilliant blue R-250, 45\% (v/v) ethanol, and 10\% (v/v) acetic acid]. To assay the EndoN-mediated removal of PSA from NCAM, neurons were costained for PSA (mouse anti-PSA antibody; AbCys) and GAP-43, visualized with Alexa-conjugated goat anti-rabbit or goat anti-mouse antibodies (Invitrogen), and mounted with anti-fade mounting medium (Dako).

Micrographs of neurons were recorded by computer-assisted microscopy using a Nikon Diaphot 300 inverted microscope equipped with a Plan $20 \times$ objective (Nikon) and coupled to a video camera (Grundig Electronics). The average length of neuronal processes per cell was estimated by a stereological approach using the software package "ProcessLength" developed at the Protein Laboratory (Copenhagen, Denmark) (Rønn et al., 2000). Approximately 200 cells were analyzed for each condition in each individual experiment.

Additional micrographs were obtained using a Radiance 2000 confocal laser scanning system (Bio-Rad) attached to a Nikon Eclipse TE 200 microscope equipped with a $60 \times$ objective (Nikon).

\section{FGFR phosphorylation assay}

T-REx-293 cells stably transfected with human FGFR1, splice variant IIIc, with a C-terminal Strep II tag were seeded in $6 \mathrm{~cm}$ cell culture dishes (Nalge Nunc International) at $1.4 \times 10^{5} \mathrm{cells} / \mathrm{cm}^{2}$ in DMEM containing $10 \%$ (v/v) FCS, $2 \mathrm{~mm}$ GlutaMAX, $200 \mu \mathrm{g} / \mathrm{ml}$ hygromycin, $100 \mathrm{U} / \mathrm{ml}$ penicillin, and $100 \mu \mathrm{g} / \mathrm{ml}$ streptomycin, and grown for $30 \mathrm{~h}$. Cells were then starved overnight in medium without serum, before being treated with GDNF, Gliafin, or FGF2 for 20 min. Subsequently, cells were lysed in $300 \mu \mathrm{l}$ of lysis buffer [ $1 \%(\mathrm{v} / \mathrm{v}) \mathrm{NP}-40,2 \%(\mathrm{v} / \mathrm{v})$ Complete EDTA-free protease inhibitor mixture, and $1 \%(\mathrm{v} / \mathrm{v})$ phosphatase inhibitor mixture set III in PBS].

Tyrosine-phosphorylated proteins were immunoprecipitated by incubating $500 \mu \mathrm{g}$ of proteins from each lysate with $15 \mu \mathrm{l}$ of agarose-coupled anti-phosphotyrosine antibodies (4G10-AC; Millipore ) overnight at $4^{\circ} \mathrm{C}$. Agarose beads were washed, and bound proteins were eluted with phenylphosphate and separated by SDS-PAGE. Additionally, to assay the level of total strep-tagged FGFR, and actin expression, $60 \mu \mathrm{g}$ of proteins from each original lysate were separated by SDS-PAGE. Proteins were then transferred to Immobilon-P membranes (Millipore) and stained using anti-StrepII tag antibody (QIAGEN) in 5\% (w/v) nonfat dry milk or anti-actin antibody (Sigma-Aldrich), followed by incubation with appropriate HRP-conjugated antibodies (Dako) in 5\% (w/v) nonfat dry milk. The immune complexes were visualized by chemiluminescence (SuperSignal West Dura Extended Duration substrate; Pierce Biotechnology) using a GeneGnome (Syngene) and the accompanying GenTools software.

\section{SPR analysis}

Binding analysis was performed using a BIAcore 2000 instrument (BIAcore). Analysis was performed at $25^{\circ} \mathrm{C}$ using HBS-EP [ 10 mM HEPES, pH 7.4, $150 \mathrm{~mm} \mathrm{NaCl}, 3 \mathrm{~mm}$ EDTA, 0.005\% (v/v) Surfactant P20] as running buffer. Proteins were immobilized on a CM4 sensor chip at a flow rate of $5 \mu \mathrm{l} / \mathrm{min}$ using an amine coupling kit (BIAcore). The chip was activated with $35 \mu \mathrm{l}$ of activation solution. For analysis of binding of various recombinant NCAM proteins to GDNF, $\sim 7 \mu \mathrm{l}$ of $1.7 \mu \mathrm{M}$ recombinant human GDNF in $10 \mathrm{~mm}$ sodium acetate, $\mathrm{pH}$ 5.0, was injected over the chip, yielding an immobilization level of $\sim 1000$ resonance units (RU). For analysis of binding of the GDNF-derived peptides to various recombinant NCAM proteins, the following volumes and concentrations were used for immobilization: $40 \mu \mathrm{l}$ of $20 \mu \mathrm{M}$ NCAM Ig1-3 in $10 \mathrm{~mm}$ sodium acetate, $\mathrm{pH} 4.0,30 \mu \mathrm{l}$ of $0.5 \mu \mathrm{M}$ NCAM Ig1-5 without VASE in $10 \mathrm{~mm}$ sodium acetate, $\mathrm{pH} 4.0$, and $30 \mu \mathrm{l}$ of $2.4 \mu \mathrm{M}$ NCAM Ig3-5 without VASE in $10 \mathrm{~mm}$ sodium acetate, $\mathrm{pH}$ 4.0. The resulting immobilization level was $\sim 1000$ RU for each of the NCAM recombinant proteins. The chip was then blocked with $35 \mu$ l of blocking solution. Proteins/peptides tested for binding were diluted in HBS-EP buffer and injected over the chip at a flow rate of $10-30 \mu \mathrm{l} / \mathrm{min}$. Regeneration was performed with $150 \mathrm{~mm}$ $\mathrm{NaCl}$ containing $12.5 \mathrm{~mm} \mathrm{NaOH}$ or $10 \mathrm{~mm}$ glycine- $\mathrm{HCl}, \mathrm{pH}$ 1.5. The curves corresponding to the difference between the binding to the protein in interest and the binding to a blank chip (activated and deactivated) were used for analysis. All curves were further referenced by subtracting appropriate control curves obtained by injecting HBS-EP buffer alone. Curves were then analyzed by nonlinear curve fitting using the software package BIAevaluation, version 4.1 (BIAcore). Curves were either fitted using a 1:1 interaction model, in which case $K_{\mathrm{D}}$ was calculated as $k_{\mathrm{d}} / k_{\mathrm{a}}$, or evaluated using steady-state affinity analysis.

\section{Statistical analysis}

Statistical analysis was performed using Student's $t$ test or one-way repeated-measures ANOVA followed by either Dunnett's or Tukey's post hoc tests using either GraphPad Prism, version 4.02 (GraphPad), or SAS, version 9.1 (SAS Institute). Results are expressed as mean \pm SEM. Unless otherwise stated, untreated controls are set to $100 \%$ for all neurite outgrowth data. The corresponding average absolute length per neuron for controls is provided in the figure legends. Significance levels are indicated as follows: ${ }^{\star,+, \#} p<0.05 ;^{\star *,++, \# \#} p<0.01 ;^{\star * *,+++, \# \#} p<0.001$.

\section{Results}

\section{GDNF induces differentiation of hippocampal neurons via NCAM}

Within recent years, GDNF has been clearly shown to signal independently of Ret (Poteryaev et al., 1999; Trupp et al., 1999; Pezeshki et al., 2001), and NCAM can function as an alternative receptor for GDNF (Paratcha et al., 2003), the latter interaction leading to induction of neurite outgrowth when hippocampal neurons are grown on a GDNF substrate. We studied whether soluble GDNF induces the neurite outgrowth response in primary neurons in the absence or presence of trans-homophilic NCAM interactions.

To distinguish NCAM-mediated effects of GDNF from Retmediated effects, we chose to work with hippocampal neurons that express NCAM but not Ret (Doherty et al., 1992b; Nosrat et al., 1997; Yu et al., 1998; Golden et al., 1999; Kiryushko et al., 2003). We confirmed by Western blotting that hippocampal neurons did not express Ret but did express NCAM and the coreceptor GFR $\alpha 1$ (supplemental Fig. S1, available at www.jneurosci.org as supplemental material). 
A

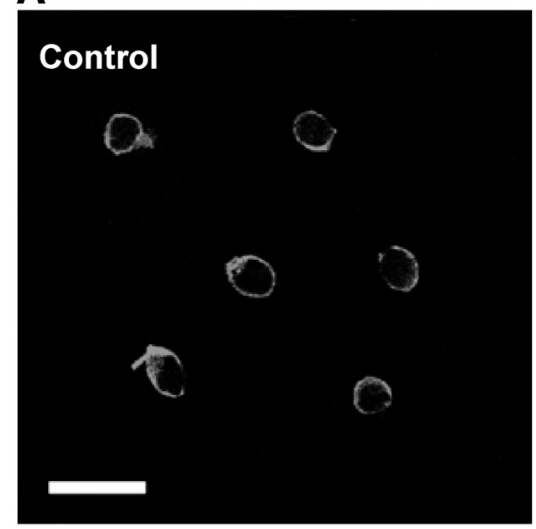

B

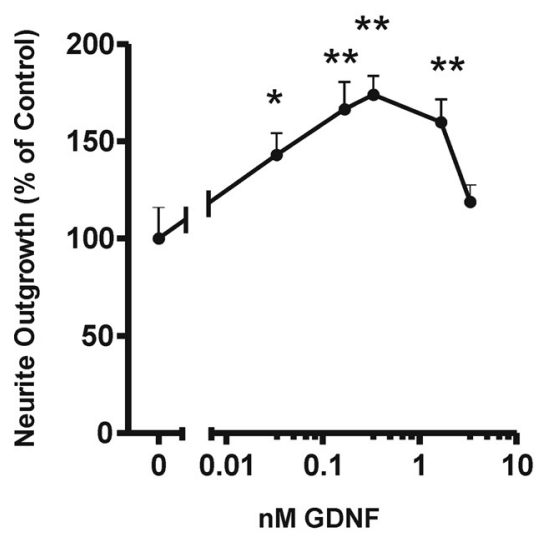

D

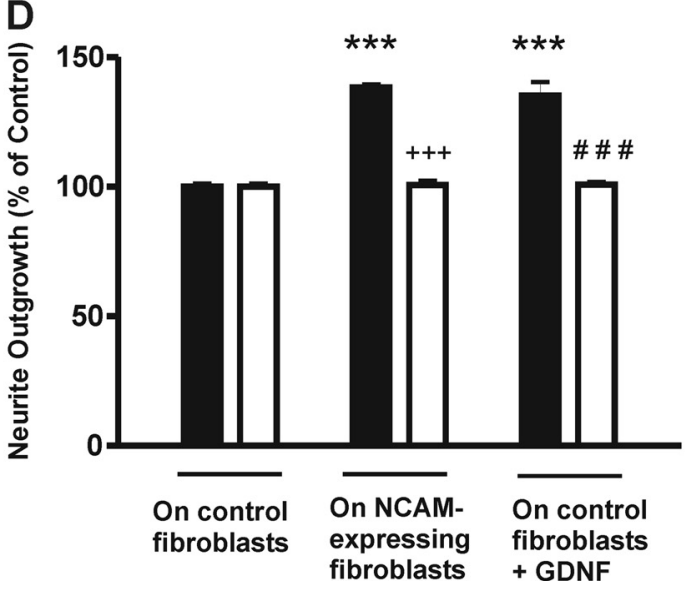

Figure 1. GDNF induces neurite outgrowth from hippocampal neurons via NCAM. Hippocampal neurons were grown for $24 \mathrm{~h}$ in the presence of $0,0.03,0.17,0.3,1.7$, and $3.3 \mathrm{~nm}$ GDNF. $A$, Confocal micrographs of representative untreated neurons and neurons treated with $0.3 \mathrm{~nm}$ GDNF. Scale bar, $25 \mu \mathrm{m}$. $\boldsymbol{B}-\boldsymbol{D}$, Quantification of neurite outgrowth from hippocampal neurons. $\boldsymbol{B}$, Neurons grown on permanox plastic. ${ }^{*}$ Compared with untreated control on plastic $(8.83 \pm 1.42$ $\mu m ; n=9$ ). C, Neurons grown on monolayers of control fibroblasts (filled circles) or NCAM-expressing fibroblasts (open circles). ${ }^{*}$ Compared with untreated control on control fibroblasts $(39.56 \pm 0.69 \mu \mathrm{m} ; n=8) .{ }^{+}$Compared with control on NCAM-expressing fibroblasts. D, Hippocampal neurons transfected with either control shRNA plasmid (black bars) or NCAM shRNA (white bars) were grown on monolayers of control fibroblasts or NCAM-expressing fibroblasts for $24 \mathrm{~h}$ in the absence or presence of $0.3 \mathrm{~nm} \mathrm{GDNF} .{ }^{*}$ Compared with control neurons (control shRNA, control fibroblasts, without GDNF; $30.02 \pm$ $0.36 \mu \mathrm{m} ; n=4) .{ }^{+}$Compared with NCAM-stimulated neurons (control shRNA, NCAM-expressing fibroblasts, without GDNF). "Compared with GDNF-stimulated neurons (control shRNA, control fibroblasts, with GDNF). Error bars indicate SEM.

Hippocampal neurons were grown at a low density (to avoid any cell-cell interactions) on plastic, and the ability of GDNF to induce neurite outgrowth was evaluated by incubating neurons with different concentrations of GDNF for $24 \mathrm{~h}$. When compared with control cultures of neurons grown in medium alone, GDNF induced an increase in neurite outgrowth (Fig. 1A). Quantification of GDNFinduced neurite outgrowth revealed a bell-shaped dose-response relationship (Fig. $1 B$ ). Statistical analysis showed that the overall neuritogenic effect of GDNF was statistically significant $\left(F_{(5,48)}=7.78\right.$; $p<0.0001)$. The maximal effect of GDNF, $\sim 175 \%$ increase in neurite outgrowth relative to control, was obtained with $0.3 \mathrm{~nm} \mathrm{GDNF}(10 \mathrm{ng} / \mathrm{ml})$. Dunnett's multiple-comparison post hoc test showed that concentrations of GDNF from 0.03 to $1.7 \mathrm{~nm}$ produced levels of neurite outgrowth significantly different from those of control cultures (Fig. 1B), thereby substantiating that GDNF in solution can induce neurite outgrowth in hippocampal neurons.

The ability of NCAM to induce neurite extension via homophilic interactions is well known (for review, see Walmod et al., 2004). Homophilic NCAM interactions occur both between NCAM molecules on the same cell (interactions in cis) and between NCAM molecules on juxtaposed cells (interactions in trans). Both types of interactions have been shown to be necessary for neurite outgrowth induced by homophilic NCAM interactions (Soroka et al., 2003). A well established system for studying the effects of trans-homophilic NCAM binding on neurite outgrowth is a coculture system, in which neurons are grown on top of a monolayer of either NCAM-negative control cells or NCAMexpressing cells (Doherty et al., 1989).

It is unknown whether homophilic NCAM interactions inhibit or promote the ability of GDNF to induce neurite outgrowth. To investigate this, we incubated neurons, grown in coculture with either control fibroblasts or NCAMexpressing fibroblasts, with different concentrations of GDNF. As shown in Figure $1 C$, filled circles, GDNF induced significant neurite outgrowth with a bell-shaped dose-response relationship in hippocampal neurons grown on control fibroblasts $\left(F_{(5,42)}=26.64 ; p<0.0001\right)$, supporting the hypothesis that homophilic NCAM trans-interactions are not needed for GDNF-induced neuritogenesis. The maximal effect $(\sim 155 \%$ of control) was obtained with $0.3 \mathrm{~nm}$ GDNF. All tested concentrations of GDNF induced neurite outgrowth significantly different from control (Fig. 1C, filled circles). As expected, homophilic NCAM interactions led to an induction of neuritogenesis when neurons were grown on a monolayer of NCAM-expressing fibroblasts. The level of NCAM-induced neurite outgrowth (control cultures 


\section{A GAP-43}
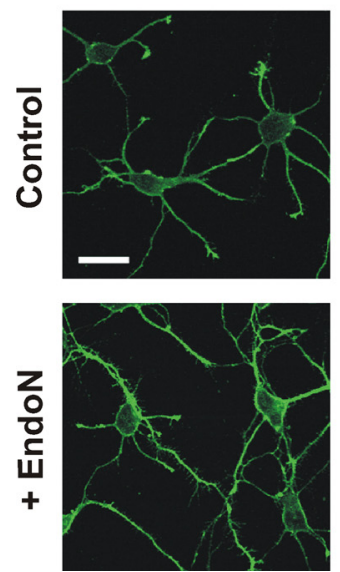

PSA-NCAM
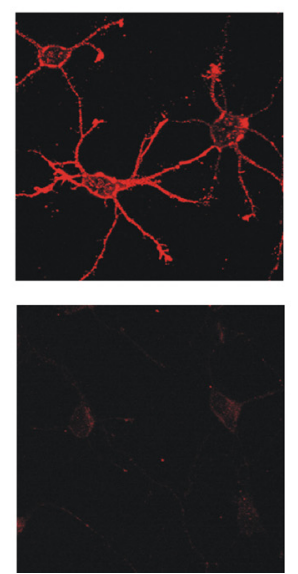

Merged

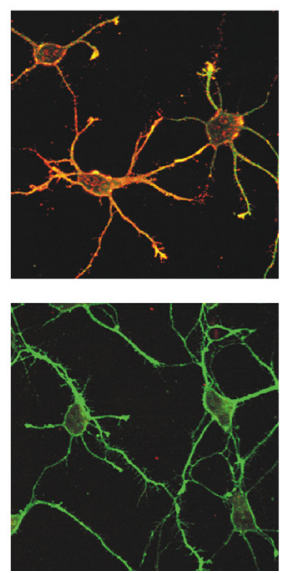

B

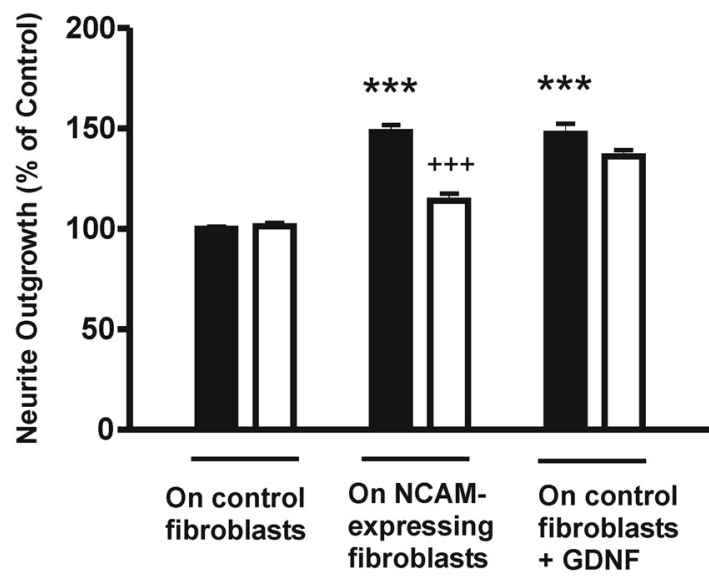

Figure 2. Polysialylation of NCAM is not required for GDNF-induced neurite outgrowth. Hippocampal neurons were grown on monolayers of control fibroblasts or NCAM-expressing fibroblasts for $24 \mathrm{~h}$ in the absence or presence of $0.3 \mathrm{~nm}$ GDNF without (black bars) or with (white bars) $60 \mathrm{~nm}$ EndoN. $\boldsymbol{A}$, Confocal micrographs of untreated neurons (control) and EndoN-treated neurons (+EndoN) stained for GAP-43 (green) and PSA (red). Scale bar, $25 \mu \mathrm{m} . B$, Quantification of the effect of PSA removal on GDNF-induced neurite outgrowth. ${ }^{*}$ Compared with control neurons (control fibroblasts, without EndoN and GDNF; $40.73 \pm 0.44 \mu \mathrm{m} ; n=5$ ). ${ }^{+}$Compared with NCAM-stimulated neurons (NCAM-expressing fibroblasts, without EndoN and GDNF). Error bars indicate SEM.

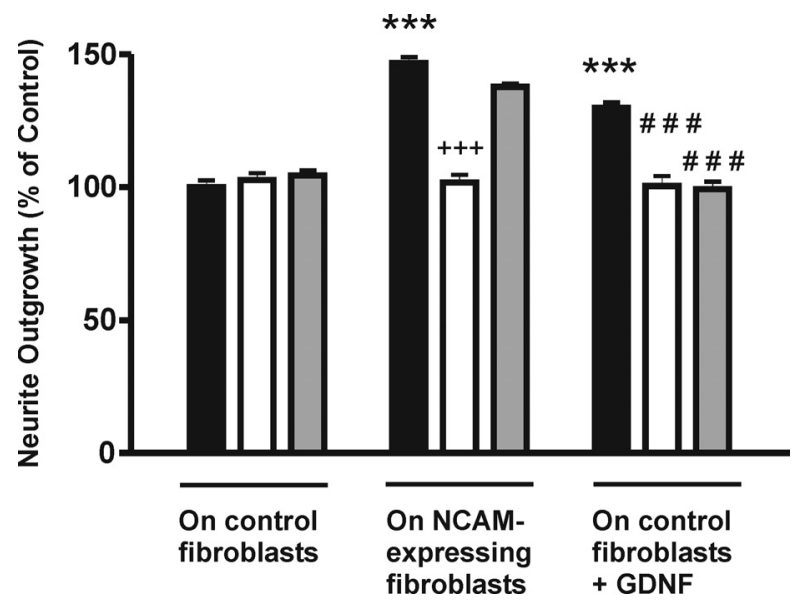

Figure 3. Both NCAM-140 and NCAM-180 mediate GDNF-induced neurite outgrowth. Hippocampal neurons transfected with control plasmid (black bars), cyt NCAM-180 plasmid (white bars), or cyt NCAM-140 plasmid (gray bars) were grown on monolayers of control fibroblasts or NCAM-expressing fibroblasts for $18 \mathrm{~h}$ in the absence or presence of $0.3 \mathrm{~nm}$ GDNF. * Compared with control neurons (control plasmid, control fibroblasts, without GDNF; $35.06 \pm 0.88 \mu \mathrm{m} ; n=7-8) .{ }^{+}$Compared with NCAM-stimulated neurons (control plasmid, NCAM-expressing fibroblasts, without GDNF). " Compared with GDNFstimulated neurons (control plasmid, control fibroblasts, with GDNF). Error bars indicate SEM.

grown on NCAM-expressing fibroblasts) was $\sim 140 \%$ of the neurite outgrowth in control cultures grown on control fibroblasts $(p<0.001)$. Incubation of neurons grown on NCAM-expressing fibroblasts with different concentrations of GDNF revealed that GDNF could induce additional neurite extension in these neurons (Fig. 1C, open circles), indicating that the effects of GDNF and homophilic NCAM interactions on neurite outgrowth are additive. The neuritogenic effect of GDNF exhibited a bellshaped dose-response relationship for neurons grown on NCAM-expressing fibroblasts, and the overall effect of GDNF was statistically significant $\left(F_{(5,42)}=12.22 ; p<0.0001\right)$. The maximal effect was $\sim 177 \%$ of the level of neurite outgrowth in control cultures of neurons grown on control fibroblasts and was obtained with $0.3 \mathrm{~nm}$ GDNF. Additionally, all tested concentrations of GDNF, with the exception of $3 \mathrm{~nm}$, induced neurite out- growth significantly different from that of control cultures of neurons grown on NCAM-expressing fibroblasts (Fig. 1C).

To conclude, these experiments show that GDNF, independently of Ret, can induce neurite outgrowth in hippocampal neurons and that this effect does not depend on, but rather can occur in the presence of, homophilic NCAM interactions.

The neurite outgrowth effect on hippocampal neurons stimulated with GDNF may have been mediated by the alternative GDNF receptor NCAM. However, the existence of additional alternative GDNF receptors has recently been indicated (Pozas and Ibáñez, 2005; Cao et al., 2008). We therefore used shRNA to knock down NCAM expression in hippocampal neurons to investigate the requirement of NCAM for GDNF-induced hippocampal neurite outgrowth (Fig. $1 D$ ). Neurons were transfected with either a plasmid encoding shRNA for NCAM or control shRNA (Hansen et al., 2007). To confirm the knockdown of NCAM expression, the transfected neurons were stained for NCAM. Transfection with shRNA for NCAM clearly reduced NCAM expression (supplemental Fig. S2A, available at www. jneurosci.org as supplemental material). Transfected neurons were seeded on control fibroblasts and stimulated with $0.3 \mathrm{nM}$ GDNF. As a positive control for the effect of knocking down NCAM expression, neurons were also seeded on NCAMexpressing fibroblasts. Neurons transfected with control shRNA and grown on NCAM-expressing fibroblasts exhibited a significant increase in neurite outgrowth $(\sim 140 \%$ of control) compared with neurons transfected with control shRNA and grown on control fibroblasts (Fig. 1D, compare columns 3, 1) $(p<$ 0.001). As expected, knockdown of NCAM expression significantly inhibited neurite outgrowth induced by trans-homophilic NCAM interactions (Fig. $1 D$, compare columns 4,3$)(p<$ $0.001)$. When neurons transfected with control shRNA were stimulated with $0.3 \mathrm{~nm}$ GDNF, neurite outgrowth was significantly stimulated to $\sim 135 \%$ of control cultures (Fig. $1 D$, compare columns 5, 1) $(p<0.001)$. Knockdown of NCAM expression led to significant inhibition of GDNF-induced neurite outgrowth (Fig. 1D, compare columns 6,5$)(p<0.001)$, resulting in levels that were not significantly different from controls unstimulated with GDNF. In contrast, neurite outgrowth induced independently of NCAM by the metallothionein-derived peptide EmtinB (Ambjørn et al., 2008) was unaffected by the 

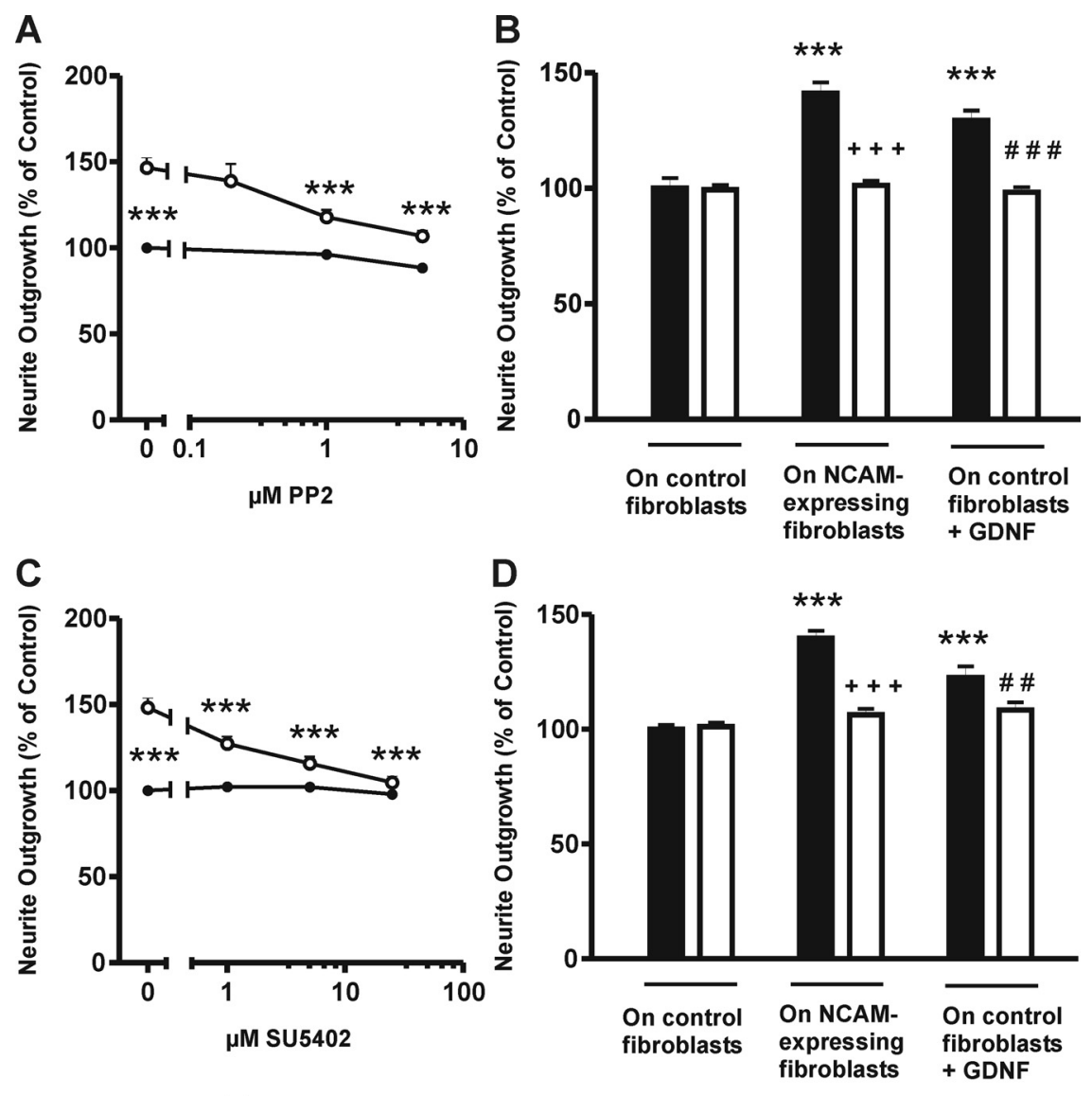

E

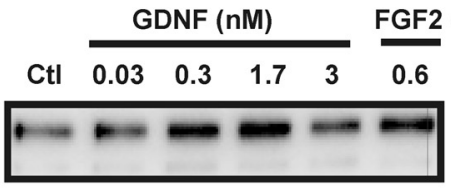

FGFR1-strep

$\beta$-actin

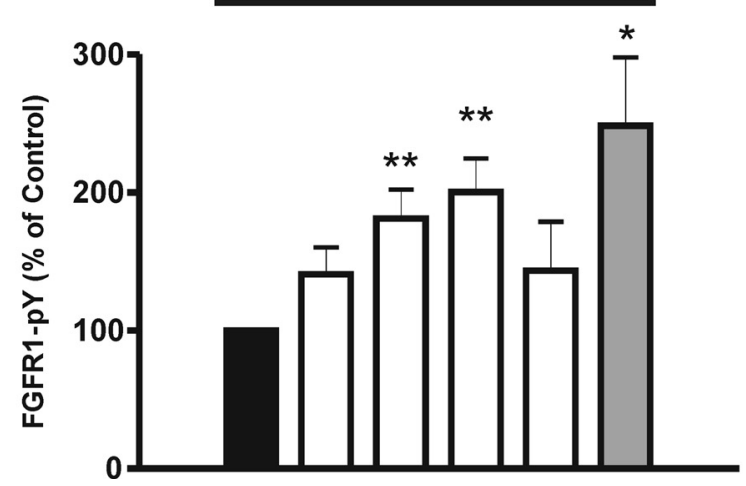

Figure 4. GDNF-induced NCAM-signaling involves both Fyn and FGFR. The importance of Fyn $(\boldsymbol{A}, \boldsymbol{B})$ and FGFR $(\boldsymbol{C}, \boldsymbol{D})$ for GDNF-induced neurite outgrowth was studied. $A, C$, Hippocampal neurons were grown on monolayers of control fibroblasts for $24 \mathrm{~h}$ in the absence (filled circles) or presence (open circles) of $0.3 \mathrm{~nm}$ GDNF and treated with the Src family kinase inhibitor PP2 ( 0.2 , 1 , and $5 \mu \mathrm{M})(\boldsymbol{A})$ or the FGFR inhibitor SU5402 $(1,5$, and $25 \mu \mathrm{M})(\boldsymbol{C})$. Neurons grown without PP2 or SU5402 served as controls. ${ }^{*}$ Compared with GDNF-stimulated neurons ( $\boldsymbol{A}$, with GDNF, without PP2: $58.28 \pm 1.79 \mu \mathrm{m}, n=4-8$; $\boldsymbol{C}$, with GDNF, without SU5402: $59.68 \pm 1.40 \mu \mathrm{m}, n=4-6$ ). $\boldsymbol{B}$, Hippocampal neurons transfected with either control plasmid (black bars) or dnFyn plasmid (white bars) were grown on monolayers of control fibroblasts or NCAM-expressing fibroblasts for $24 \mathrm{~h}$ in the absence or presence of $0.3 \mathrm{~nm}$ GDNF. * Compared with control neurons (control plasmid, control fibroblasts, without GDNF; $30.42 \pm 1.33 \mu \mathrm{m}$; $n=3) .{ }^{+}$Compared with NCAM-stimulated neurons (control plasmid, NCAM-expressing fibroblasts, without GDNF). ${ }^{~}$ Compared with GDNF-stimulated neurons (control plasmid, control fibroblasts, with GDNF). D, Hippocampal neurons transfected with either knockdown of NCAM (supplemental Fig. S2 $B$, available at www.jneurosci.org as supplemental material). To conclude, these results confirm that neurite outgrowth induced by GDNF is indeed mediated via NCAM in hippocampal neurons.

NCAM polysialylation is not required for GDNF-induced neuritogenesis

The most prominent posttranslational modification of NCAM is the addition of PSA to NCAM Ig5. Polysialylation affects multiple functions of NCAM and is known to promote neurite outgrowth induced by homophilic NCAM interactions (Doherty et al., 1990). NCAM polysialylation is most prominent during embryonic and early postnatal life (for review, see Bonfanti, 2006). Accordingly, NCAM on the embryonic hippocampal neurons used in the experiments described above is highly polysialylated (Fig. $2 \mathrm{~A}$; supplemental Fig. S1B, available at www. jneurosci.org as supplemental material). PSA can be enzymatically removed from NCAM by EndoN. Staining for PSA on hippocampal neurons incubated with $60 \mathrm{~nm}$ EndoN for $24 \mathrm{~h}$ showed that EndoN treatment strongly reduced NCAM polysialylation (Fig. 2A). Moreover, when neurons where incubated with 60 $\mathrm{nM}$ EndoN and grown on either control fibroblasts or NCAM-expressing fibroblasts, a significant reduction of NCAMinduced neurite outgrowth was observed (Fig. $2 B$, compare columns 4,3$)(p<$ $0.001)$, confirming that PSA-NCAM promotes neuritogenesis. In contrast, removal of PSA did not significantly alter GDNFinduced neurite outgrowth. The level of neurite outgrowth in neurons treated with $60 \mathrm{~nm}$ EndoN and stimulated with $0.3 \mathrm{~nm}$ GDNF ( $135 \%$ of control) was not significantly different from the level of neu-

$\leftarrow$

control plasmid (black bars) or dnFGFR plasmid (white bars) were grown on monolayers of control fibroblasts or NCAMexpressing fibroblasts for $18 \mathrm{~h}$ in the absence or presence of 0.3 nm GDNF. *Compared with control neurons (control plasmid, control fibroblasts, without GDNF; $34.19 \pm 0.64 \mu \mathrm{m} ; n=5$ ). ${ }^{+}$Compared with NCAM-stimulated neurons (control plasmid, NCAM-expressing fibroblasts, without GDNF). "Compared with GDNF-stimulated neurons (control plasmid, control fibroblasts, with GDNF). E, T-REx-293 cells were stimulated with the indicated concentrations of GDNF or FGF2 and assayed for FGFR phosphorylation. Top, Representative immunoblots of phosphorylated FGFR1 (pY-FGFR1) and total level of streptagged FGFR1 (FGFR1-strep). Bottom, Quantification of FGFR phosphorylation in unstimulated cells (black bar), GDNFtreated cells (white bars), and FGF2-treated cells (gray bar). FGFR phosphorylation is expressed as a percentage of unstimulated control, which is set to $100 \%(n=5-8)$. ${ }^{*} \mathrm{Com}$ pared with unstimulated control. Error bars indicate SEM. 
rite outgrowth in neurons stimulated with GDNF in the absence of EndoN ( $\sim 145 \%$ of control) (Fig. $2 B$, compare columns 6,5$)(p>0.05)$. These results indicate that PSA is not required for GDNF-induced neurite outgrowth mediated by NCAM. Consistent with this, we could not detect any binding of the PSA analog colominic acid to GDNF when tested by SPR analysis (see supplemental Fig. S3A, available at www.jneurosci.org as supplemental material), indicating that there is no direct interaction between GDNF and PSA and supporting the notion that PSA is not involved in NCAM-mediated GDNF-induced neurite outgrowth.

\section{GDNF-induced neurite outgrowth involves both NCAM-140 and NCAM-180}

We next studied which isoform of NCAM, NCAM-140 or NCAM-180, mediates the neuritogenic response to GDNF. We transfected hippocampal neurons with plasmids encoding the cytoplasmic domain of either NCAM-180 or NCAM-140, which have been shown specifically to abrogate NCAM180- and NCAM-140-mediated neurite outgrowth, respectively (Kolkova et al., 2000b; Korshunova et al., 2007). When control-transfected neurons were grown on NCAM-expressing fibroblasts, a significant increase in neurite outgrowth was observed compared with cells grown on control fibroblasts (Fig. 3, compare columns 4, 1) $(p<$ $0.001)$. Consistent with previous findings (Korshunova et al., 2007), uncoupling of NCAM-180 signaling was found to significantly inhibit NCAM-induced neurite outgrowth (Fig. 3, compare columns 5, 4) $(p<0.001)$, whereas uncoupling of NCAM-140 signaling had no effect (Fig. 3, compare columns 6,4$)(p>0.05)$. Stimulation with $0.3 \mathrm{nM}$ GDNF of controltransfected neurons grown on control fibroblasts significantly induced neurite outgrowth to $\sim 130 \%$ relative to unstimulated neurons (Fig. 3, compare columns 7, 1$)(p<0.001)$. In contrast to NCAM-induced neurite outgrowth, GDNF-induced neurite outgrowth was significantly inhibited both by uncoupling of NCAM-180 and NCAM-140 signaling, respectively (Fig. 3, compare columns 8,7 , and 9, 7, respectively) ( $p<0.001)$. These results demonstrate that both isoforms of NCAM are involved in the transduction of GDNF signaling via NCAM and point to a difference in the mechanisms underlying neurite outgrowth induced by homophilic NCAM interactions and by GDNF via NCAM.

\section{GDNF-induced signaling downstream of NCAM involves both Fyn and FGFR}

NCAM does not possess any intracellular catalytic activity. Therefore, we investigated how the binding of GDNF to NCAM translates into an intracellular signal. Two tyrosine kinases have been shown to be essential for neurite outgrowth induced by homophilic NCAM interactions. These are the Src family nonreceptor protein tyrosine kinase (SFK) Fyn and the receptor protein

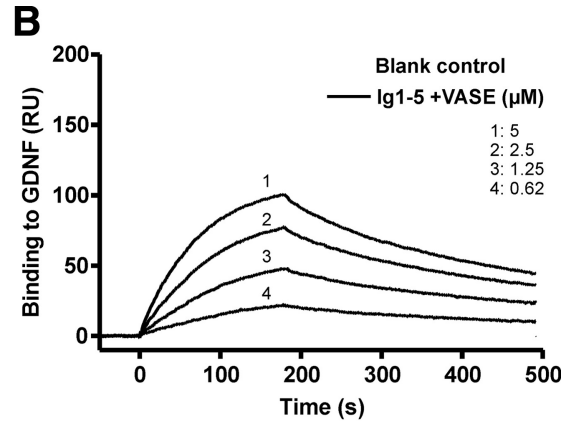

D

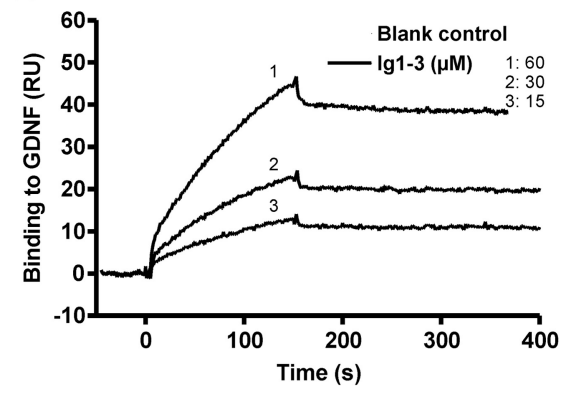

E

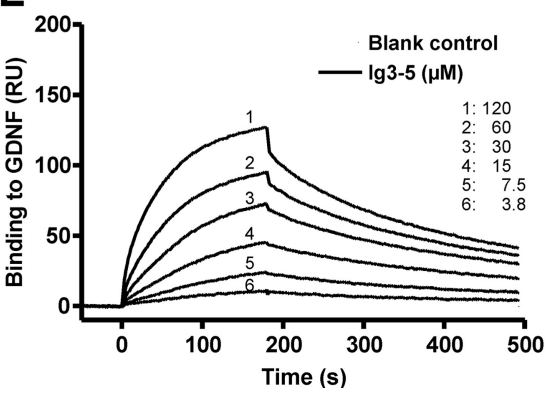$$
\text { . }
$$

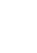

Figure 5. A binding site for GDNF is located within NCAM Ig3. GDNF was immobilized on a sensorchip, and various recombinant proteins were injected into the chip at a flow rate of $30 \mu \mathrm{l} / \mathrm{min}$. $\boldsymbol{A}-\boldsymbol{E}$, Representative binding curves for the binding of GFR $\alpha 1(\boldsymbol{A})$, Ig1-5 + VASE (B), Ig1-5 - VASE (C), Ig1-3 (D), and lg3-5 (E) to GDNF. Injections were initiated at time 0 and lasted for $270 \mathrm{~s}(\boldsymbol{A})$, $180 \mathrm{~s}(\boldsymbol{B}, \boldsymbol{C}, \boldsymbol{E})$, and $150 \mathrm{~s}(\boldsymbol{D})$. Concentrations of injected proteins are indicated in each panel.

tyrosine kinase FGFR (Beggs et al., 1994; Saffell et al., 1997; Kolkova et al., 2000a; Niethammer et al., 2002). Thus, we tested whether these kinases are also involved in signaling leading to GDNF-induced neurite outgrowth via NCAM.

Neurons were grown on control fibroblasts and stimulated with $0.3 \mathrm{~nm}$ GDNF for $24 \mathrm{~h}$ in the absence or presence of different concentrations of the pharmacological SFK inhibitor PP2. Stimulation with GDNF in the absence of PP2 significantly increased neurite outgrowth compared with control (Fig. $4 A)(p<0.001)$. Incubation of unstimulated neurons with PP2 did not significantly alter the basal level of neurite outgrowth compared with unstimulated neurons grown in the absence of PP2. In contrast, both 1 and $5 \mu \mathrm{M}$ PP2 significantly reduced GDNF-induced neurite outgrowth (Fig. $4 A)(p<0.001$ in both cases), and inhibition was almost complete with $5 \mu \mathrm{M}$ PP2. Another SFK inhibitor, SU6656, also significantly inhibited GDNF-induced neurite outgrowth without affecting the basal level of neurite outgrowth (supplemental Fig. S4, available at www.jneurosci.org as supplemental material). These data indicate that a SFK is involved in signaling leading to GDNF-induced neurite outgrowth. Because Fyn is the SFK known to mediate signaling downstream of NCAM, we assumed that Fyn was the SFK involved in NCAMmediated GDNF-induced neurite outgrowth. To investigate this 
Table 1. Rate and affinity constants for the binding of GFR $\alpha 1$ and various recombinant NCAM proteins to GDNF

\begin{tabular}{lll}
\hline Analyte & $k_{\mathrm{a}}\left(\mathrm{M}^{-1} \mathrm{~s}^{-1}\right)$ & $K_{\mathrm{d}}\left(\mathrm{s}^{-1}\right)$ \\
\hline GFR $\alpha 1$ & $\mathrm{n} . \mathrm{d}$. & $\mathrm{K} . \mathrm{d}$. \\
$\lg 1-5+$ VASE & $2.3 \times 10^{3} \pm 1.2 \times 10^{2}$ & $3.0 \times 10^{-3} \pm 1.5 \times 10^{-4}$ \\
$\lg 1-5-$ VASE & $2.7 \times 10^{3} \pm 2.1 \times 10^{2}$ & $3.1 \times 10^{-3} \pm 1.8 \times 10^{-4}$ \\
$\lg 1-3$ & $1.1 \times 10^{2} \pm 4.1 \times 10^{0}$ & $2.0 \times 10^{-4} \pm 5.1 \times 10^{-5}$ \\
$\lg 3-5$ & $2.3 \times 10^{2} \pm 2.7 \times 10^{-6} \pm 8.2 \times 10^{-8}$ & $1.2 \times 10^{-6} \pm 1.2 \times 10^{-7}$ \\
\hline
\end{tabular}

GDNF was immobilized on a sensorchip by amine coupling and GFR $\alpha 1$, or various recombinant NCAM proteins were injected into the chip. The equilibrium binding levels were used to evaluate the $K_{D}$ for the binding of GFR $\alpha 1$ to $G D N F$ ( $n=$ 2). For the binding of recombinant NCAM proteins to GDNF, $k_{\mathrm{a}}$ and $k_{\mathrm{d}}$ were evaluated by nonlinear curve fitting using nonequilibrium binding curves, and $K_{\mathrm{D}}$ was calculated as $k_{\mathrm{d}} / k_{\mathrm{a}}(n=4-6)$. All constants are expressed as mean $\pm S E M$. n.d., Not determined.

assumption, neurons were transfected with a plasmid encoding a dominant-negative version of Fyn (dnFyn) (Mariotti et al., 2001). Control-transfected hippocampal neurons grown on NCAM-expressing fibroblasts exhibited a significant increase in neurite outgrowth compared with neurons grown on control fibroblasts (Fig. $4 B$, compare columns 3,1$)(p<0.001)$. Neurite outgrowth induced by homophilic NCAM interactions was significantly inhibited when Fyn activity was inhibited by expression of dnFyn (Fig. $4 B$, compare columns 4,3$)(p<0.001)$. Expression of dnFyn in neurons stimulated with $0.3 \mathrm{~nm}$ GDNF and grown on control fibroblasts also led to significant inhibition of neurite outgrowth compared with GDNF-stimulated neurite outgrowth in control-transfected neurons (Fig. $4 \mathrm{~B}$, compare columns 6,5$)(p<0.01)$. To conclude, these data indicate that Fyn is involved in GDNF-induced neurite outgrowth in hippocampal neurons, thereby confirming previous findings (Paratcha et al., 2003).

To evaluate the involvement of FGFR in GDNF-induced neurite outgrowth, hippocampal neurons, which are known to express FGFR (Neiiendam et al., 2004), were grown on control fibroblasts and stimulated with $0.3 \mathrm{nM}$ GDNF in the absence or presence of different concentrations of the pharmacological FGFR inhibitor SU5402. In the absence of SU5402, GDNF significantly induced neurite outgrowth (Fig. $4 C)(p<0.001)$. Basal levels of neurite outgrowth were not affected by incubation with SU5402. In contrast, incubation with SU5402 dose-dependently reduced the GDNF-induced neurite outgrowth response, and the reduction was significant for all concentrations of SU5402 applied (Fig. 4C) ( $p<0.001$ in all cases), and $25 \mu \mathrm{M}$ SU5402 essentially abrogated GDNF-induced neurite outgrowth. These results indicate that FGFR also is involved in signaling leading from GDNF-NCAM interactions to neurite outgrowth. Next, we applied an alternative test for FGFR involvement. Neurons were transfected with a plasmid encoding a kinase-deficient dominant-negative version of FGFR (dnFGFR) (Saffell et al., 1997). When control-transfected hippocampal neurons were grown on NCAM-expressing fibroblasts, a significant increase in neurite outgrowth was observed compared with neurons grown on control fibroblasts (Fig. $4 D$, compare columns 3,1$)(p<$ 0.001). Inhibition of FGFR activation by expression of dnFGFR led to a significant inhibition of neurite outgrowth induced by homophilic NCAM interactions (Fig. 4D, compare columns 4, 3) $(p<0.001)$. Expression of dnFGFR in neurons stimulated with $0.3 \mathrm{~nm}$ GDNF and grown on control fibroblasts also led to a significant reduction of neurite outgrowth compared with GDNF-stimulated neurite outgrowth in control-transfected neurons (Fig. $4 D$, compare columns 6,5$)$ ( $p<0.01$ ), further supporting the conclusion that FGFR plays a role in GDNF-induced neurite outgrowth in hippocampal neurons.

Subsequently, we investigated whether GDNF was able to induce FGFR phosphorylation. We used T-REx-293 cells stably transfected with FGFR1. T-REx-293 cells express NCAM-140 (supplemental Fig. S5, available at www.jneurosci.org as supplemental material) and could thus be responsive to GDNF. Stimulating T-REx-293 cells with different concentrations of GDNF led to a significant increase in the level of FGFR phosphorylation compared with unstimulated control cells (Fig. 4E, compare white columns, black column). GDNF-induced FGFR phosphorylation exhibited a bell-shaped dose-response relationship and maximal effect ( $\sim 200 \%$ of control) was obtained with $1.7 \mathrm{nM}$ GDNF. For comparison, stimulation with $0.6 \mathrm{~nm}$ FGF2 increased the level of FGFR phosphorylation to $\sim 250 \%$ of the control (Fig. 4E, gray column). Thus, these data support the interpretation that GDNF-induced signaling via NCAM involves FGFR activation.

In summary, our results indicate that GDNF-induced signaling resulting in hippocampal neurite outgrowth appears to share some of the same pathways as the signaling involved in neurite outgrowth induced by trans-homophilic NCAM interactions.

\section{A binding site for GDNF is located within the third Ig module of NCAM}

The structural basis for the interaction between GDNF and NCAM was investigated, focusing on the interaction between GDNF and the Ig modules of NCAM. We applied an SPR-based approach and tested the binding of a number of recombinant NCAM proteins in solution to immobilized GDNF. As a positive control, we first tested the binding of GDNF to its cognate receptor, GFR $\alpha 1$, using a GFR $\alpha 1$-Fc chimera. The resulting binding response is shown in Figure $5 A$. The affinity $\left(K_{\mathrm{D}}\right)$ for the GDNFGFR $\alpha 1$ interaction was calculated by steady-state analysis and found to be $9.8 \mathrm{nM}$ (Table 1 ).

NCAM mRNA is subjected to different forms of alternative splicing, one of which results in NCAM subtypes that are distinguished by the absence or presence of the so-called VASE sequence in the Ig4 module. The presence of the VASE sequence has been shown to have a negative effect on neurite outgrowth mediated by homophilic NCAM interactions (Doherty et al., 1992a; Liu et al., 1993; Saffell et al., 1994). Recombinant NCAM Ig1-5 proteins with or without the VASE sequence were tested for their binding to GDNF. Both forms bound to GDNF (Fig. $5 B, C$ ), indicating that one or more binding sites for GDNF are localized within the Ig modules of NCAM. The rate constants $k_{\mathrm{a}}$ and $k_{\mathrm{d}}$ and the affinity constant $K_{\mathrm{D}}$ for the binding of Ig1-5 with and without VASE, respectively, to GDNF, were determined (Table 1). Pairwise comparisons of $k_{\mathrm{a}}, k_{\mathrm{d}}$, and $K_{\mathrm{D}}$ of GDNF binding to Ig1-5 with and without VASE did not reveal any significant differences between the binding parameters $(p>0.18, p>0.66$, and $p>$ 0.54 , respectively). These results indicate that the VASE sequence does not affect the binding of NCAM to GDNF.

To determine in which part of the five Ig modules of NCAM the binding site for GDNF is localized, we subsequently made use of two recombinant proteins, NCAM Ig1-3 and a VASE-negative version of NCAM Ig3-5, which in an overlapping manner cover 
all five Ig modules. Both recombinant proteins were found to bind to GDNF (Fig. 5D,E; Table 1). The fact that both Ig1-3 and Ig3-5 bound to GDNF indicates that both constructs contain one or more binding sites for GDNF. One explanation could be that the binding site for GDNF is located in Ig3, the only module that is common to the two recombinant proteins. Alternatively, multiple binding sites may be distributed between several Ig modules.

Subsequently, a recombinant protein encompassing Ig1-2 of NCAM was tested, but no binding to GDNF could be shown (supplemental Fig. S3B, available at www. jneurosci.org as supplemental material), indicating that Ig3 is necessary for the binding of NCAM to GDNF and supporting the hypothesis that a binding site for GDNF exists within Ig3. However, when using recombinant Ig3 alone, no binding could be detected (supplemental Fig. S3C, available at www. jneurosci.org as supplemental material), suggesting that Ig3 alone is not sufficient for the binding of NCAM to GDNF (but see next paragraph) and implying the existence of a multimodular binding site. Attempts to produce a folded protein encompassing only NCAM Ig4-5 were unsuccessful, and we were therefore unable to investigate the potential presence of additional binding sites within these two modules.

An alternative interpretation of the aforementioned data is that the affinity of the binding between NCAM Ig3 and GDNF might be too low for detection by SPR. Because of the homophilic interaction between Ig1 and Ig2 of NCAM (Kiselyov et al., 1997; Atkins et al., 1999; Jensen et al., 1999; Kasper et al., 2000), Ig1-2, Ig1-3, and Ig1-5 will dimerize in solution. Studies of Ig3-5 by dynamic light scattering and gel filtration have indicated that this protein dimerizes in solution (data not shown). The fact that these proteins exist as dimers raises the possibility that they bind bivalently to GDNF. In contrast, Ig3 in solution does not form dimers (Kiselyov et al., 1997; Atkins et al., 2001; Soroka et al., 2003) and may thus only bind monovalently to GDNF. Bivalent binding greatly enhances affinity; the $K_{\mathrm{D}}$ for a bivalent interaction theoretically equals the square of the $K_{\mathrm{D}}$ for the corresponding monomer (Kiselyov et al., 1997). Given that the $K_{\mathrm{D}}$ for the interaction between NCAM Ig1-5 and GDNF was found to be $\sim 1$ $\mu \mathrm{M}$, the $K_{\mathrm{D}}$ for binding of Ig3 to GDNF should be $\sim 1 \mathrm{~mm}$ (i.e., too low to be detected by SPR analysis). To test this possibility, we used a version of Ig1-3 (Ig1-3 mut) (Soroka et al., 2003) that is unable to form dimers. This construct did not show any binding to GDNF (supplemental Fig. S3D, available at www.jneurosci.org as supplemental material), presumably because the affinity of this interaction is below the detection limit of the SPR system, thus supporting the hypothesis of a bivalent interaction between NCAM and GDNF.

To clarify whether Ig3 is sufficient for the binding of NCAM to GDNF, we added recombinant Ig3 to cultures of hippocampal neurons grown on control fibroblasts in the presence of $0.3 \mathrm{nM}$ GDNF. If Ig3 has a binding site for GDNF, it should compete with NCAM on the neurons for the binding of GDNF, thus resulting in a decrease in GDNF-induced neurite outgrowth. As shown in Figure $6 A$, soluble NCAM Ig3 indeed dose-dependently inhibited GDNF-induced neurite outgrowth but had no effect on the

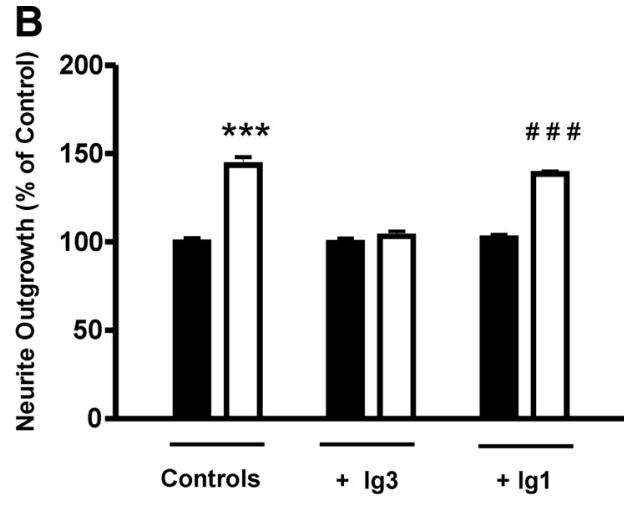
Ig3 module is sufficient for binding of NCAM to GDNF. As a control for the specificity of the inhibitory effect of Ig3, we also applied another Ig module, Ig1. The addition of Ig1 to hippocampal neurons stimulated with $0.3 \mathrm{~nm}$ GDNF did not affect GDNFinduced neurite outgrowth, whereas the same concentration of Ig3 abolished GDNF-induced neurite outgrowth (Fig. 6B). This finding supports the specificity of the observed interaction between NCAM Ig3 and GDNF.

\section{Identification of a binding site for NCAM within GDNF}

To determine which part in GDNF interacts with NCAM, we designed three peptides covering putative NCAM-binding sequences of GDNF. These peptides essentially cover each of the finger regions, which have previously been shown to be important for binding of GDNF to GFR $\alpha 1$ (Eketjäll et al., 1999; Baloh et al., 2000; Parkash et al., 2008) and the heel region (Fig. 7A). We termed these peptides Finger 1 peptide, Finger 2 peptide, and Gliafin (term derived from glial cell line-derived neurotrophic factor and the suffix "in"), respectively. We then used SPR analysis to evaluate the binding of these peptides in solution to immobilized NCAM Ig1-5 without VASE. We found that the Gliafin peptide covering the heel region of GDNF bound to NCAM (Fig. $7 B$, green curve), whereas the two peptides from the finger regions of GDNF did not (Fig. 7B, red and blue curves). A scrambled version of Gliafin showed considerably less binding to NCAM than the original peptide, demonstrating the specificity of the interaction (Fig. $7 B$, gray curve). These results suggest that a binding site for NCAM is located within the heel region of GDNF. Conversely, the finger regions of GDNF do not appear to be involved in the binding of GDNF to NCAM. Steady-state affinity analysis was applied to determine the affinity for the binding of Gliafin to Ig1-5, which was found to be in the low micromolar range (Table 2 ).

Additional experiments revealed that Gliafin, like GDNF, also bound to Ig1-3 and Ig3-5, whereas the peptides covering the finger regions of GDNF did not (Fig. 7C,D). Again, the binding of Gliafin to Ig1-3 and Ig3-5 appeared to be specific, demonstrated by the much lower binding of the scrambled peptide. Steady-state affinity analysis showed that the affinities for the binding of Gliafin to Ig1-3 and Ig3-5, respectively, also were in the low micro- 
A

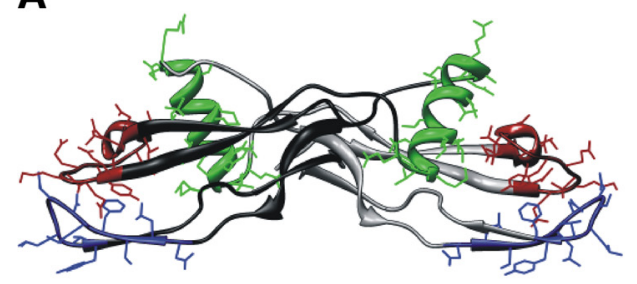

Finger 1 peptide: 48-LNVTDLGLGYETKEE-62

Finger 2 peptide: $110-D L S F L D D S L V Y-120$

Gliafin: 76-ETMYDKILKNLSRSR-90
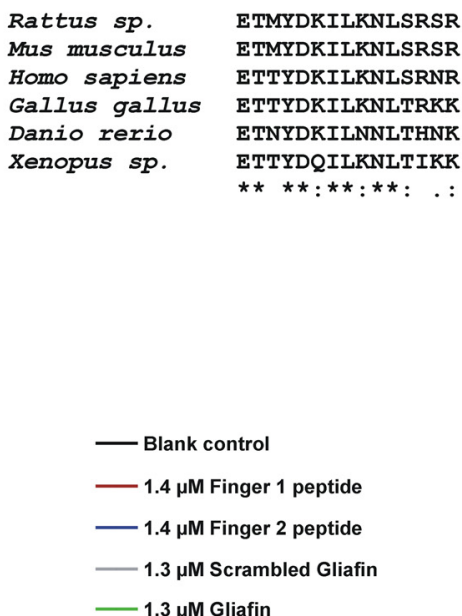
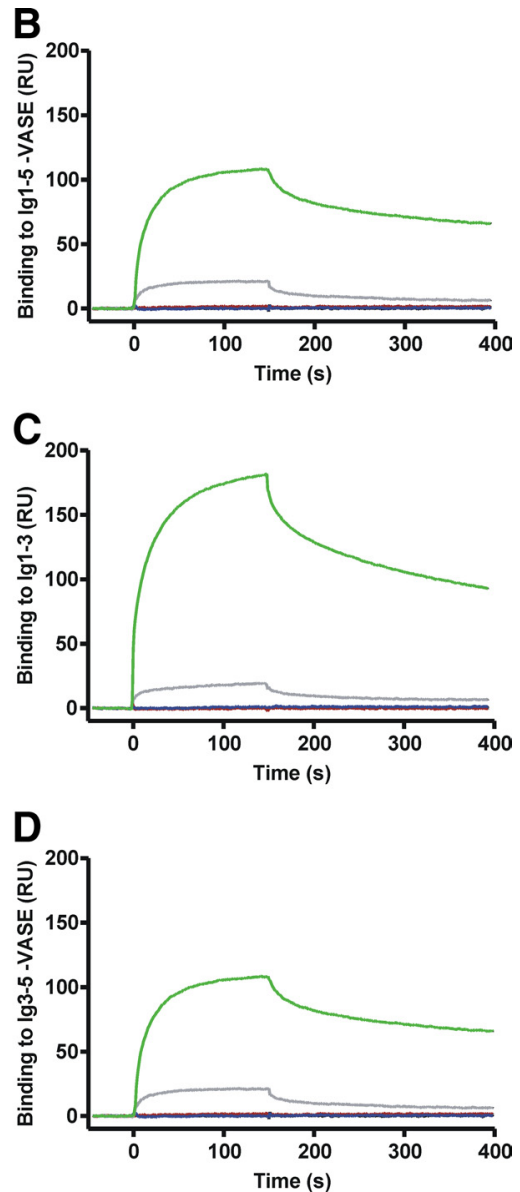

Figure 7. Identification of a binding site for NCAM within GDNF. A, Ribbon structure of the GDNF homodimer (one monomer in black, the other in gray) showing the position of the three GDNF-derived peptides. Gliafin is indicated in green, and the Finger 1 and Finger 2 peptides are shown in red and blue, respectively. Shown below in corresponding colors are the peptide sequences. Residue numbering is based on the $x$-ray structure of rat GDNF (PDB 1AGQ). The figure was prepared using the University of California, San Francisco Chimera package from the Resource for Biocomputing, Visualization, and Informatics at the University of California, San Francisco. Interspecies comparisons of the Gliafin peptide sequence from Rattus sp. (ENSRNOP00000017222), Mus musculus (ENSMUSP00000022744), Homo sapiens (ENSP00000339703), Gallus gallus (ENSGALP00000005887), Danio rerio (ENSDARP00000058462), and Xenopus sp. (ENSXETP00000010369). Peptide sequences were obtained from the Ensembl genome browser (www.ensembl.org; Ensemble peptide ID numbers given above), and sequence alignment was performed with the T-COFFEE multiple sequence alignment program (www.ebi.ac.uk). “*” indicates identical, ":" indicates conserved substitution, and "." indicates semiconserved substitution. $\boldsymbol{B}-\boldsymbol{D}$, Representative binding curves showing the binding of GDNF-derived peptides to various recombinant NCAM proteins. NCAM Ig1-5 - VASE $(\boldsymbol{B})$, Ig1-3 (C), and Ig3-5 - VASE (D) were immobilized on a sensorchip, and peptides at the indicated concentrations were injected into the chip at a flow rate of $10 \mu \mathrm{l} / \mathrm{min}$. Injections were initiated at time 0 and lasted for $150 \mathrm{~s}$.

Table 2. Affinity constants for the binding of Gliafin to various recombinant NCAM proteins

\begin{tabular}{ll}
\hline Immobilized ligand & $K_{\mathrm{D}}$ (M) \\
\hline $\lg 1-5$ & $6.0 \times 10^{-7} \pm 1.7 \times 10^{-8}$ \\
Ig1-3 & $1.1 \times 10^{-6} \pm 2.5 \times 10^{-8}$ \\
$\lg 3-5$ & $3.5 \times 10^{-7} \pm 2.6 \times 10^{-8}$ \\
\hline
\end{tabular}

NCAM Ig1-5 - VASE, Ig1-3, and Ig3-5 - VASE were immobilized on a sensorchip by amine coupling, and Gliafin $(0.08,0.16,0.32,0.64,1.28,2.56$, and $5.12 \mu \mathrm{m})$ was injected into the chip at a flow rate of $10 \mu \mathrm{l} / \mathrm{min}$ for $25 \mathrm{~min}$. The equilibrium binding levels from two independent experiments were used to evaluate the $K_{D}$, which is expressed as mean \pm SEM.

molar range (Table 2). Together, these data indicate the existence of a binding site for NCAM within the heel region of GDNF. Furthermore, the data reveal a soluble peptide sequence derived from GDNF that binds to the NCAM ectodomain. An interspecies comparison of the Gliafin peptide sequence shows that the
Gliafin sequence shares $80 \%$ similarity between the six investigated species (Fig. 7A, bottom).

The heel region of GDNF is formed by an $\alpha$-helix. Displaying the residues of the $\alpha$-helix on a helical wheel (supplemental Fig. S6A, available at www.jneurosci.org as supplemental material) clearly shows that this $\alpha$-helix is an amphipathic helix that is enriched in hydrophobic side chains on one side and in hydrophilic side chains on the other. In the rat GDNF dimer (PDB 1AGQ), the $\alpha$-helix of one GDNF molecule extends in such a way that the side with hydrophobic residues is facing a cluster of hydrophobic residues located in the finger regions of the second GDNF molecule (supplemental Fig. S6B, available at www.jneurosci.org as supplemental material). Thus, the hydrophobic side of the Gliafin sequence is involved in the formation of the GDNF dimer, whereas the hydrophilic side is free for other interactions. Consistent with the $\alpha$-helical conformation of the Gliafin sequence within GDNF, circular dichroism spectroscopy indicated that the Gliafin peptide also has a predominantly $\alpha$-helical conformation (supplemental Fig. S6C, available at www.jneurosci.org as supplemental material).

\section{The heel region peptide Gliafin is a functional GDNF mimetic targeting NCAM}

Having identified a binding sequence for NCAM within GDNF, we tested whether the corresponding peptide had any biological effect as determined by its ability to induce neurite outgrowth in vitro. Hippocampal neurons were grown at low density on plastic and stimulated with different concentrations of the Gliafin peptide for $24 \mathrm{~h}$. A dramatic increase in neurite outgrowth was seen in Gliafintreated cultures compared with neurite outgrowth in the control culture, whereas no apparent increase was observed in cultures treated with a scrambled version of Gliafin (Fig. 8A). Quantification of Gliafin-induced neurite outgrowth revealed a bell-shaped dose-response relationship, with a maximal effect of $\sim 800 \%$ of control being obtained at a peptide concentration of $0.13 \mu \mathrm{M}$ (Fig. $8 \mathrm{~B}$, green curve). Significant stimulation of neurite outgrowth was observed at concentrations from 0.04 to $0.4 \mu \mathrm{M}(p<0.01)$. These results suggest that residues within the heel region of GDNF are responsible for the interaction between GDNF and NCAM leading to induction of neurite outgrowth. Conversely, no neurite outgrowth was observed after the addition of the two peptides covering the finger regions of GDNF (Fig. $8 \mathrm{~B}$, red and blue curves), indicating that these regions are not involved in NCAM-mediated GDNF-induced neurite outgrowth. Furthermore, when hippocampal neurons were grown on plastic for $24 \mathrm{~h}$ in the presence of $0.4 \mu \mathrm{M}$ peptide, neurite outgrowth 

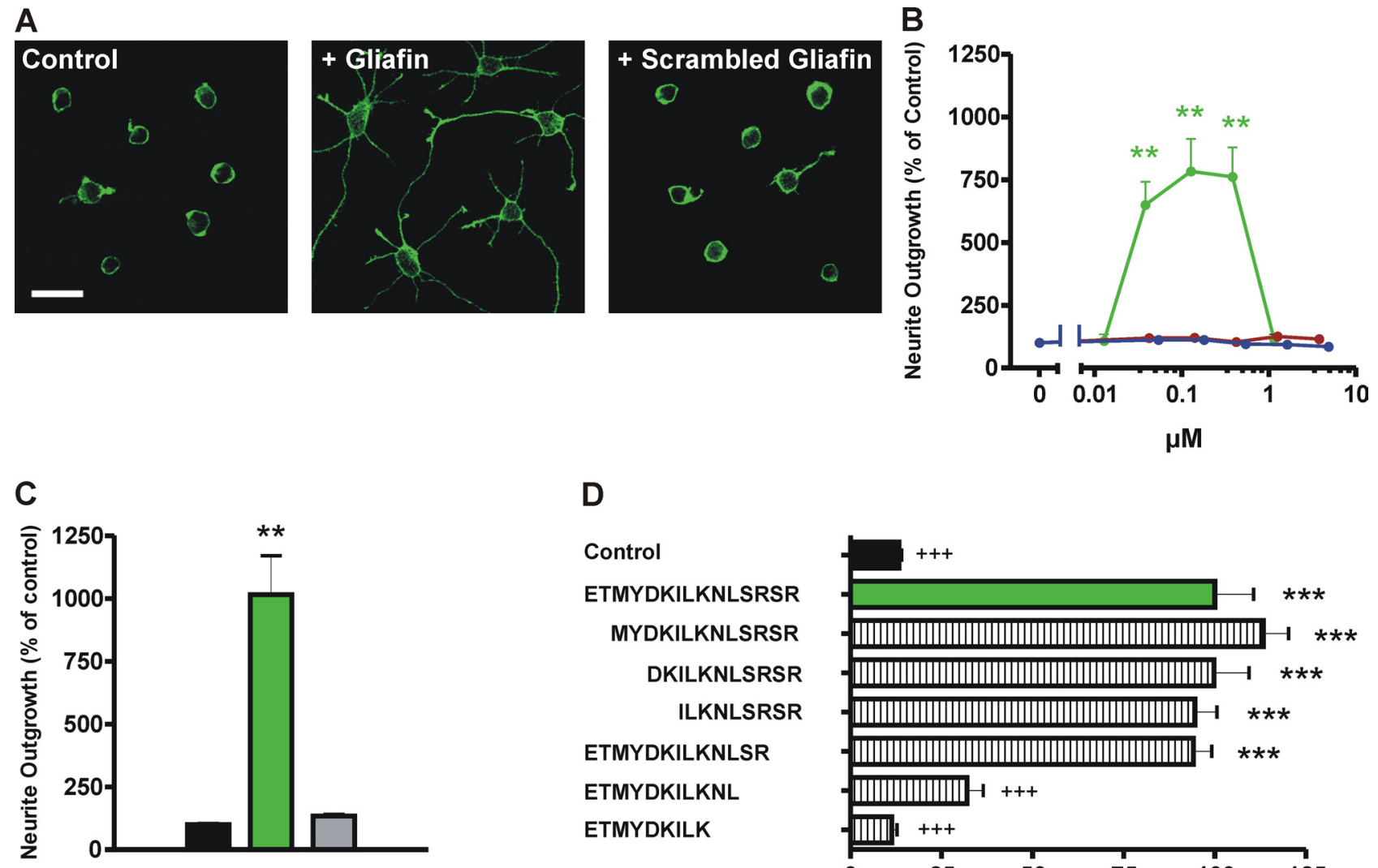

D
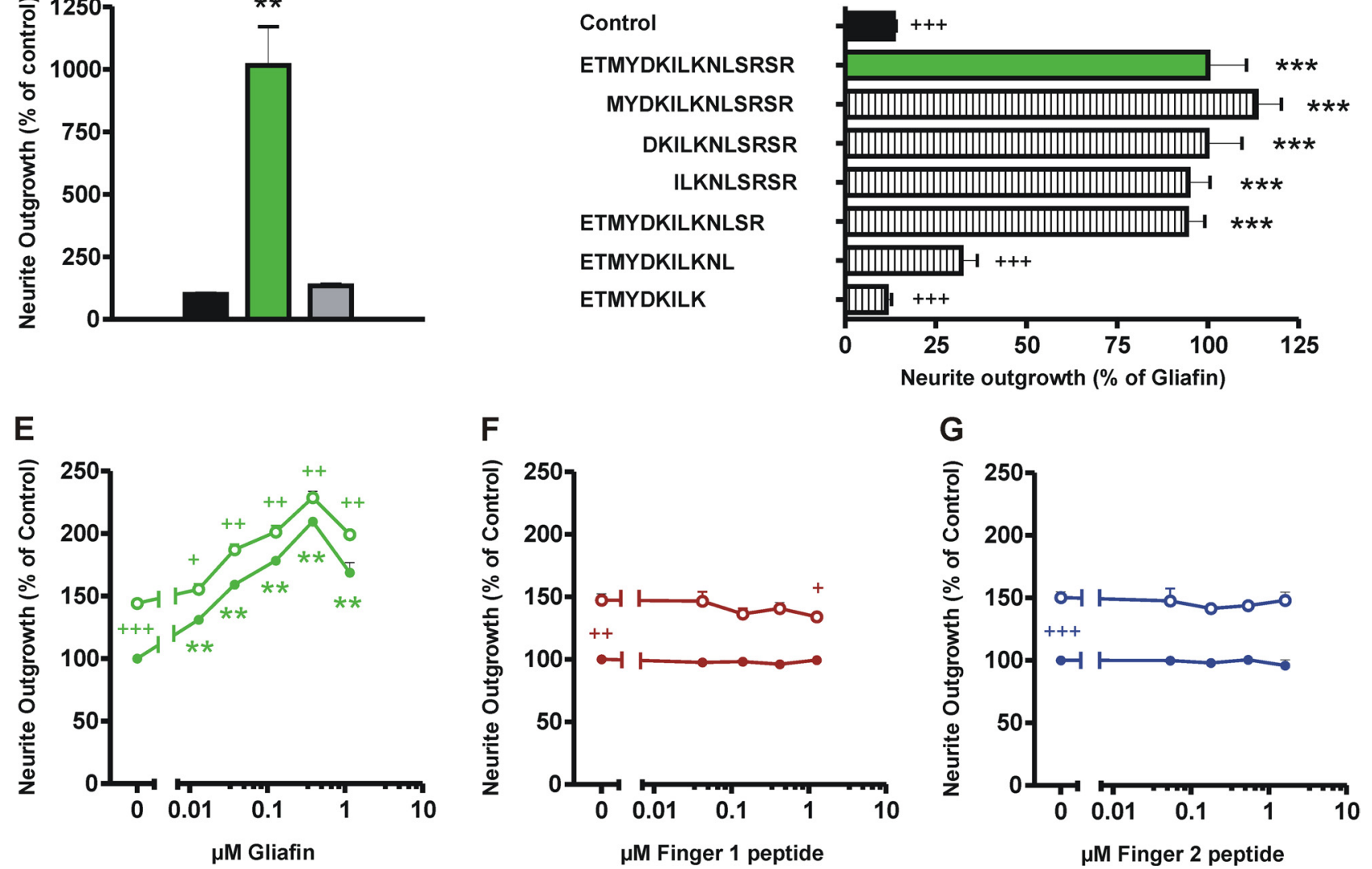

Figure 8. The heel region peptide, Gliafin, induces neurite outgrowth from hippocampal neurons. $A$, Confocal micrographs of representative untreated control neurons and neurons treated with either Gliafin or scrambled Gliafin (both at $0.4 \mu \mathrm{m}$ ). Scale bar, $25 \mu \mathrm{m}$. $\boldsymbol{B}-\boldsymbol{D}$, Quantification of neurite outgrowth from hippocampal neurons grown for $24 \mathrm{~h}$ on permanox plastic. $\boldsymbol{B}$, Neurons grown in the presence of Gliafin $(0,0.01,0.04,0.13,0.4$, and $1.2 \mu \mathrm{m}$; green circles), finger 1 peptide $(0,0.04,0.14,0.4,1.3$, and $3.8 \mu \mathrm{m}$; red circles), or finger 2 peptide $(0,0.05,0.18,0.5,1.6$, and $4.9 \mu \mathrm{M}$; blue circles). $\boldsymbol{C}$, Neurons grown in the presence of medium alone (black bar), $0.4 \mu \mathrm{m}$ Gliafin (green bar), or $0.4 \mu \mathrm{m}$ scrambled Gliafin (gray bar). $\boldsymbol{D}$, Neurons grown in the presence of medium alone (black bar), $0.4 \mu \mathrm{m}$ Gliafin (green bar; set to 100\%), or $0.4 \mu \mathrm{m}$ of one of six truncated versions of Gliafin (hatched bars). Sequences of truncated peptides are given in the figure. ${ }^{*} \mathrm{Compared} \mathrm{with}$ untreated controls ( $\boldsymbol{B}$, control for Gliafin-treated cells: $13.30 \pm 1.87 \mu \mathrm{m}, n=4$; control for finger 1-treated cells: $11.68 \pm 1.40 \mu \mathrm{m}, n=5$; control for finger 2-treated cells: $11.00 \pm 0.92 \mu \mathrm{m}$, $n=4 . C, 10.03 \pm 0.49 \mu \mathrm{m}, n=5 . \boldsymbol{D}, 11.93 \pm 0.62 \mu \mathrm{m}, n=4) .{ }^{+}$Compared with Gliafin-treated neurons. $E-G$, Quantification of neurite outgrowth from hippocampal neurons grown for $24 \mathrm{~h}$ on monolayers of control fibroblasts (filled circles) or NCAM-expressing fibroblasts (open circles) in the presence of Gliafin $(0,0.01,0.04,0.13,0.4$, and $1.2 \mu \mathrm{M})(\boldsymbol{E})$; finger 1 peptide $(0,0.04,0.14,0.4$, and $1.3 \mu \mathrm{m})(\boldsymbol{F})$; finger 2 peptide $(0,0.05,0.18,0.5$, and $1.6 \mu \mathrm{m})(\boldsymbol{G})$. ${ }^{*}$ Compared with untreated controls on control fibroblasts $(\boldsymbol{E}, 38.74 \pm 0.49 \mu \mathrm{m}, n=4 ; \boldsymbol{F}, 34.64 \pm 0.64 \mu \mathrm{m}, n=2-4$; G, $34.20 \pm 0.67 \mu \mathrm{m}, n=2-4) .{ }^{+}$Compared with controls on NCAM-expressing fibroblasts. Error bars indicate SEM.

was significantly stimulated with the Gliafin peptide (Fig. $8 C$, green column), whereas no significant effect was obtained with a scrambled version of Gliafin (Fig. $8 C$, gray column), confirming the specificity of the neuritogenic effect of Gliafin. Thus, we identified a peptide mimicking the interaction of GDNF with NCAM and the biological response resulting from this interaction.
We also tested the neuritogenic effect of a dimeric and a monomeric form of Gliafin. Circular dichroism spectroscopy indicated that both forms had a predominantly $\alpha$-helical conformation like the tetrameric form and thus were folded (supplemental Fig. S6C, available at www.jneurosci.org as supplemental material). The dimeric form of Gliafin also induced neurite out- 
growth with an efficacy similar to the tetrameric form but with a lower potency. In contrast, a monomeric form of Gliafin had no neuritogenic effect (supplemental Fig. $\mathrm{S} 6 \mathrm{D}$, available at www. jneurosci.org as supplemental material). Thus, these data indicate that the neuritogenic ability of Gliafin depends on its ability to bind more than one NCAM molecule simultaneously, suggesting that Gliafin, and thus by inference GDNF, which is a dimer, activates NCAM via a clustering mechanism.

To identify residues important for the neurite outgrowthpromoting effect of Gliafin, hippocampal neurons were grown for $24 \mathrm{~h}$ at low density on plastic and stimulated with $0.4 \mu \mathrm{M}$ of the original Gliafin peptide or one of six truncated versions of Gliafin. Significant stimulation of neurite outgrowth was obtained with the original Gliafin peptide (Fig. $8 D$, green column) $(p<0.001)$. Removal of six residues (E76-K81) from the $\mathrm{N}$-terminal end of Gliafin did not alter the level of Gliafinstimulated neurite outgrowth, indicating that these residues are not essential for the effect of Gliafin. Truncation by two residues (S89-R90) from the C terminus had no effect, whereas further removal of two C-terminal residues (S87-R88) significantly reduced neurite outgrowth to $\sim 30 \%$ of the neurite outgrowth induced by the original peptide (Fig. $8 D)(p<0.001)$. When two more residues (N85-L86) were removed from the C-terminal end, Gliafin did not retain any activity, and peptide-induced neurite outgrowth was not different from that of the unstimulated control (Fig. $8 D$ ). These results show that some or all of the four residues, N85-R88, are important for the ability of Gliafin to induce neurite outgrowth. The results indicate that the residues are important for either binding of Gliafin (and thus, by inference, GDNF) to NCAM or for retaining the proper folding of Gliafin. As seen from the sequence alignment in Figure $7 A$, bottom, N85-S87 are highly conserved within the investigated species, whereas R88 is not. This interspecies conservation suggests that the motif NLT/S may be important for Gliafin binding and/or folding.

Gliafin was also tested in the coculture system, which allows the modulation of homophilic NCAM interactions. Neurons were grown on control fibroblasts or NCAM-expressing fibroblasts and stimulated with different concentrations of Gliafin for $24 \mathrm{~h}$. Regardless of which type of fibroblast the neurons were grown on, Gliafin significantly and dose-dependently induced neurite outgrowth in a bell-shaped manner (Fig. $8 E$ ). The maximal effect $(\sim 210 \%$ of control for neurons grown on control fibroblasts and $\sim 230 \%$ for neurons grown on NCAM-expressing fibroblasts) was in both cases obtained with $0.4 \mu \mathrm{M}$ Gliafin. These data show that Gliafin, like GDNF, is able to induce neurite outgrowth both in the presence and absence of trans-homophilic NCAM interactions. The two peptides covering the finger regions of GDNF did not affect the levels of neurite outgrowth of hippocampal neurons grown on control fibroblasts or on NCAMexpressing fibroblasts (Fig. $8 F, G$ ), further supporting the hypothesis that these regions are not involved in GDNF-induced neurite outgrowth mediated by NCAM.

Knockdown of NCAM expression in hippocampal neurons by transfection with NCAM shRNA completely inhibited neurite outgrowth induced by $0.4 \mu \mathrm{M}$ Gliafin (Fig. $9 A$, compare columns $6,5)$, thereby demonstrating that NCAM is necessary for Gliafininduced neuritogenesis. These data support the hypothesis that Gliafin, like GDNF, uses NCAM as a receptor in hippocampal neurons. Moreover, when Ig3 was added to hippocampal neurons grown on control fibroblasts and stimulated with $0.4 \mu \mathrm{M}$ Gliafin, a marked dose-dependent decrease in neurite outgrowth was seen (Fig. 9B). This finding indicates that Gliafin, like GDNF,
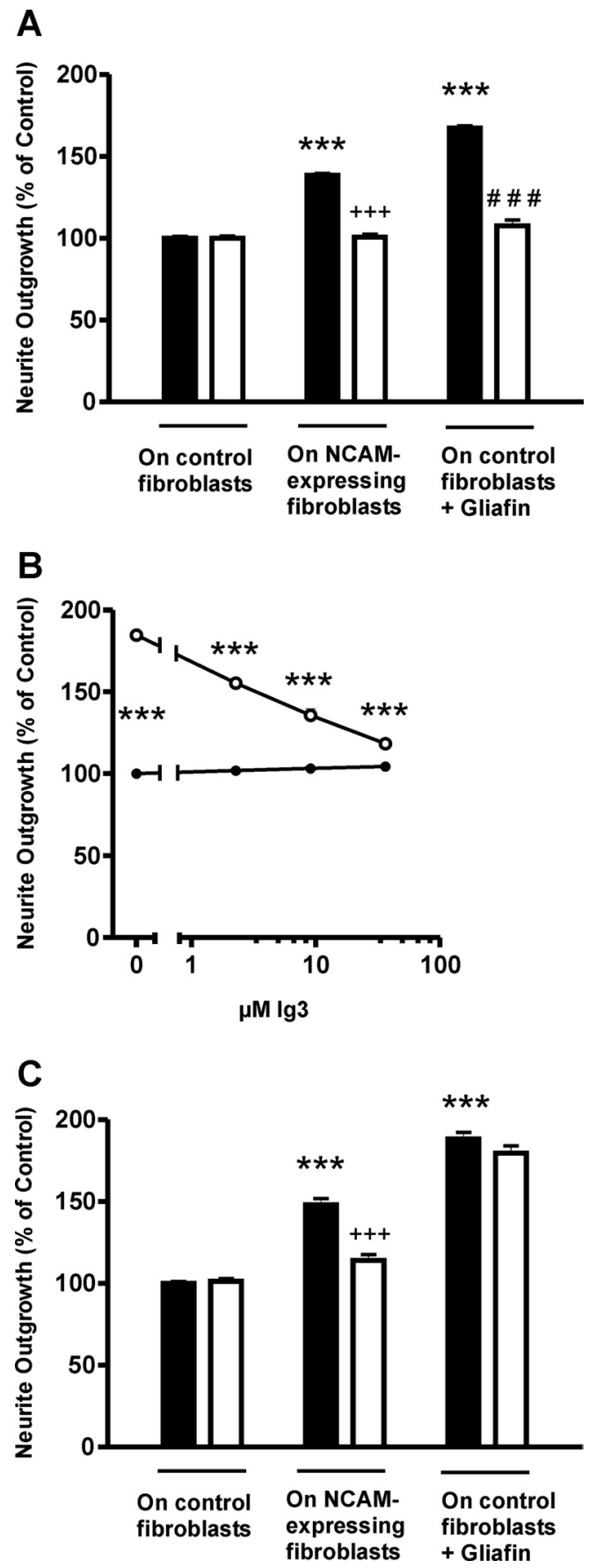

Figure 9. Gliafin-induced neurite outgrowth is mediated via NCAM but does not require polysialylated NCAM. $\boldsymbol{A}$, Hippocampal neurons transfected with either control shRNA plasmid (black bars) or NCAM shRNA (white bars) were grown on monolayers of control fibroblasts or NCAM-expressing fibroblasts for $24 \mathrm{~h}$ in the absence or presence of $0.4 \mu \mathrm{m}$ Gliafin. *Compared with control neurons (control shRNA, control fibroblasts, without Gliafin; $30.02 \pm 0.36 \mu \mathrm{m}$, $n=4) .{ }^{+}$Compared with NCAM-stimulated neurons (control shRNA, NCAM-expressing fibroblasts, without Gliafin). "Compared with Gliafin-stimulated neurons (control shRNA, control fibroblasts, with Gliafin). $\boldsymbol{B}$, Hippocampal neurons were grown on monolayers of control fibroblasts for $24 \mathrm{~h}$ in the absence (filled circles) or presence (open circles) of $0.4 \mu \mathrm{m}$ Gliafin and incubated with recombinant $\lg 3(2.3,9.1$, and $36.4 \mu \mathrm{m})$. Neurons grown without $\lg 3$ served as controls. * ${ }^{*}$ Compared with Gliafin-stimulated neurons (with Gliafin, without Ig3; $74.33 \pm 0.17$ $\mu \mathrm{m} ; n=4)$. C, Hippocampal neurons were grown on monolayers of control fibroblasts or NCAM-expressing fibroblasts for $24 \mathrm{~h}$ in the absence or presence of $0.4 \mu \mathrm{M}$ Gliafin without (black bars) or with (white bars) $60 \mathrm{~nm}$ EndoN. * Compared with control neurons (control fibroblasts, without EndoN and Gliafin; $40.73 \pm 0.44 \mu \mathrm{m} ; n=5) .{ }^{+}$Compared with NCAM-stimulated neurons (NCAM-expressing fibroblasts, without EndoN and Gliafin). Error bars indicate SEM. 
interacts with Ig3 in solution, thereby preventing Gliafin from interacting with NCAM on the neurons. Furthermore, the ability of Gliafin to induce neurite outgrowth was not significantly affected by the desialylation of NCAM by EndoN (Fig. 9C, compare columns 6,5 ), demonstrating that NCAM polysialylation is not necessary for Gliafin-induced neurite outgrowth mediated via NCAM. Thus, Gliafin closely resembles GDNF with respect to its NCAMbinding properties and neuritogenic activity, further supporting the interpretation that Gliafin represents an NCAMbinding site of GDNF.

Uncoupling of NCAM signaling by transfection of hippocampal neurons with plasmids encoding the intracellular domain of NCAM-180 and NCAM-140, respectively, suggested that both NCAM isoforms are involved in mediating Gliafininduced neurite outgrowth (Fig. 10A). Uncoupling of signaling through NCAM180 resulted in a significant decrease in neurite outgrowth induced by $0.4 \mu \mathrm{M}$ Gliafin compared with Gliafin-stimulated control-transfected neurons (Fig. 10A, compare columns 8,7$)(p<0.001)$, as did uncoupling of signaling through NCAM140 (Fig. 10A, compare columns 9, 7) $(p<0.001)$. When neurons stimulated with $0.4 \mu \mathrm{M}$ Gliafin were grown in the presence of the SFK inhibitors PP2 or SU6656, a clear dose-dependent reduction in neurite outgrowth was observed compared with Gliafin-stimulated neurons grown in the absence of PP2 or SU6656 (supplemental Fig. S7 A, B, available at www.jneurosci.org as supplemental material), and inhibition of Fyn activity by expression of dnFyn abrogated Gliafin-induced neurite outgrowth (Fig. $10 \mathrm{~B})$. These results support the hypothesis that Gliafin-induced neurite outgrowth involves signaling via the Fyn kinase. Inhibition of FGFR activity by the pharmacological FGFR inhibitor SU5402 (supplemental Fig. S7C, available at www. jneurosci.org as supplemental material), or by transfection of hippocampal neurons with a plasmid encoding dnFGFR (Fig. 10C), also caused a clear decrease in neurite outgrowth induced by $0.4 \mu \mathrm{M}$ Gliafin compared with Gliafin-stimulated control neurons (absence of SU5402 or control-transfected, respectively). These results indicate that the signaling pathways activated by Gliafin-NCAM interactions involve FGFR. Further supporting this interpretation, stimulation of FGFR1-expressing T-REx-293 cells with different concentrations of Gliafin led to a significant increase in FGFR phosphorylation compared with an unstimulated control (Fig. 10D, compare white columns, black column). Gliafin-induced FGFR phos-
D
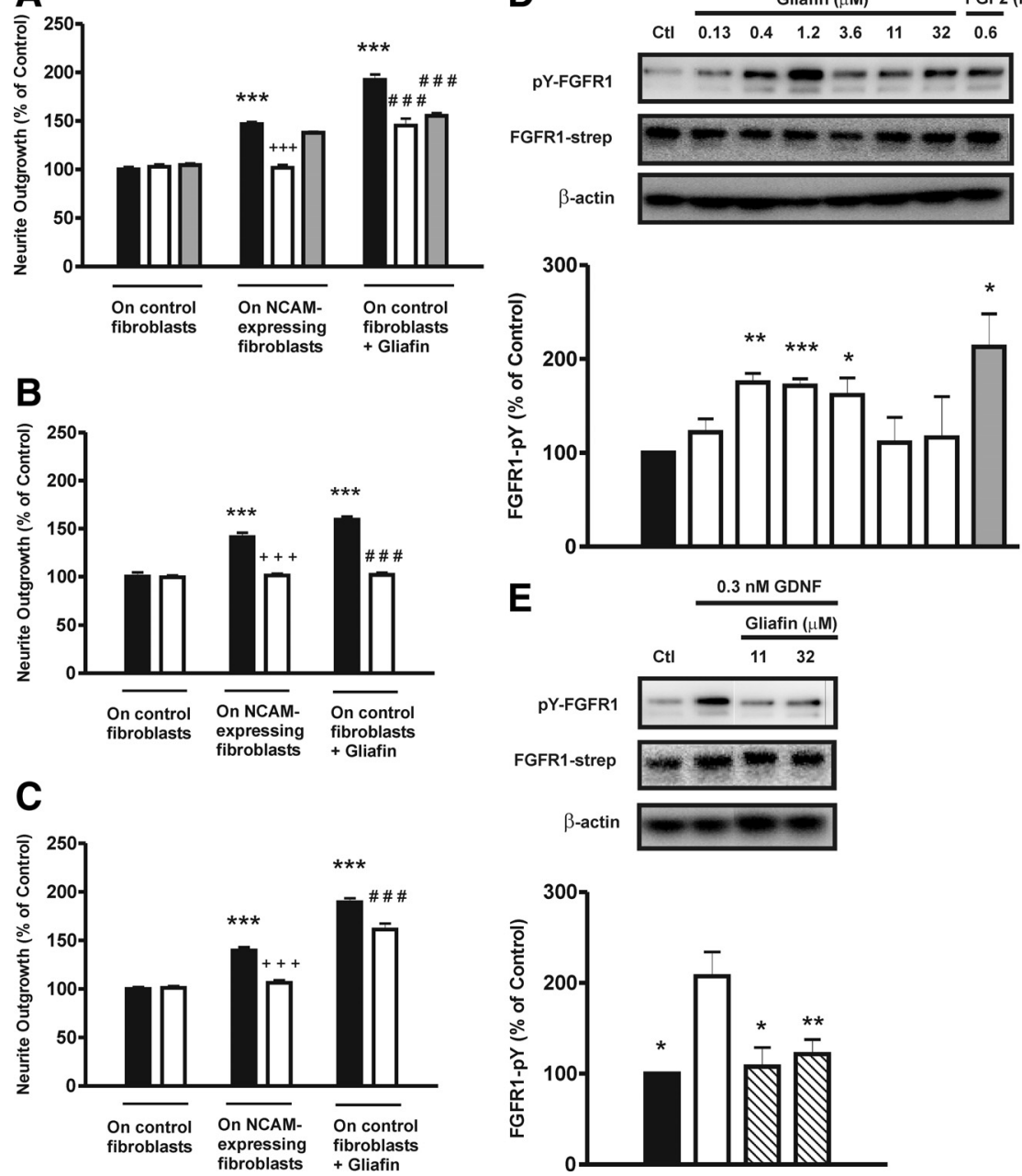

Figure 10. Gliafin-induced neurite outgrowth is mediated via NCAM-140 and NCAM-180 and involves both Fyn and FGFR signaling. A, Hippocampal neurons transfected with control plasmid (black bars), cyt NCAM-180 plasmid (white bars), or cyt NCAM-140 plasmid (gray bars) were grown on monolayers of control fibroblasts or NCAM-expressing fibroblasts for $18 \mathrm{~h}$ in the absence or presence of $0.4 \mu \mathrm{M}$ Gliafin. ${ }^{*}$ Compared with control neurons (control plasmid, control fibroblasts, without Gliafin; $35.06 \pm 0.88 \mu \mathrm{m} ; n=7-8) .{ }^{+}$Compared with NCAM-stimulated neurons (control plasmid, NCAM-expressing fibroblasts, without Gliafin). "Compared with Gliafin-stimulated neurons (control plasmid, control fibroblasts, with Gliafin). B, Hippocampal neurons transfected with either control plasmid (black bars) or dnFyn plasmid (white bars) were grown on monolayers of control fibroblasts or NCAM-expressing fibroblasts for $24 \mathrm{~h}$ in the absence or presence of $0.4 \mu \mathrm{m}$ Gliafin. ${ }^{*}$ Compared with control neurons (control plasmid, control fibroblasts, without Gliafin; $30.42 \pm 1.33 \mu \mathrm{m} ; n=3$ ). ${ }^{+}$Compared with NCAM-stimulated neurons (control plasmid, NCAM-expressing fibroblasts, without Gliafin). " Compared with Gliafin-stimulated neurons (control plasmid, control fibroblasts, with Gliafin). C, Hippocampal neurons transfected with either control plasmid (black bars) or dnFGFR plasmid (white bars) were grown on monolayers of control fibroblasts or NCAM-expressing fibroblasts for $18 \mathrm{~h}$ in the absence or presence of $0.4 \mu \mathrm{m} \mathrm{Gliafin}$. ${ }^{*}$ Compared with control neurons (control plasmid, control fibroblasts, without Gliafin; $34.19 \pm 0.64 \mu \mathrm{m} ; n=5$ ). ${ }^{+}$Compared with NCAM-stimulated neurons (control plasmid, NCAM-expressing fibroblasts, without Gliafin). " Compared with Gliafin-stimulated neurons (control plasmid, control fibroblasts, with Gliafin). D, T-REx-293 cells were stimulated with the indicated concentrations of Gliafin or FGF2 and assayed for FGFR phosphorylation. Top, Representative immunoblots of phosphorylated FGFR1 (pY-FGFR1) and total level of strep-tagged FGFR1 (FGFR1-strep). Bottom, Quantification of FGFR phosphorylation in unstimulated cells (black bar), Gliafin-treated cells (white bars), and FGF2-treated cells (gray bar). FGFR phosphorylation is expressed as percentage of unstimulated control, which is set to $100 \%(n=3-5)$. * Compared with unstimulated control. E, T-REx-293 cells were stimulated with 0.3 nм GDNF in the presence of the indicated concentrations of Gliafin and assayed for FGFR phosphorylation. Top, Representative immunoblots of phosphorylated FGFR1 (pY-FGFR1) and total level of strep-tagged FGFR1 (FGFR1-strep). Bottom, Quantification of FGFR phosphorylation in unstimulated cells (black bar), cells treated only with GDNF (white bars), and cells treated with GDNF and gliafin (hatched bars). FGFR phosphorylation is expressed as percentage of unstimulated control, which is set to $100 \%(n=4-6)$. *Compared with GDNF-stimulated control. Error bars indicate SEM.

phorylation exhibited a bell-shaped dose-response relationship. The maximal effect ( $\sim 175 \%$ of control) was obtained with $0.4 \mu \mathrm{M}$ Gliafin. FGF2 at $0.6 \mathrm{~nm}$ was included as a positive control and increased the level of FGFR phosphorylation to $\sim 220 \%$ of the control (Fig. $10 D$, gray column). 
In conclusion, the aforementioned experiments suggest that Gliafin-induced neurite outgrowth uses the same signaling pathways as those observed for GDNF-induced neurite outgrowth, thereby supporting the hypothesis that Gliafin represents the part of GDNF involved in binding to NCAM, thus resulting in GDNFinduced neurite outgrowth mediated via NCAM. Furthermore, stimulation of T-REx-293 cells with $0.3 \mathrm{~nm}$ GDNF in the presence of different concentrations of Gliafin that did not by themselves induce FGFR phosphorylation, resulted in significantly decreased GDNF-induced FGFR phosphorylation (Fig. 10 E, compare hatched columns, white column), indicating that Gliafin and GDNF compete for the same binding site and further substantiating that Gliafin represents the NCAM-binding part of GDNF.

\section{Discussion}

We investigated the structural basis for GDNF-NCAM interactions and characterized the biological effects of this interaction with regard to induction of neurite outgrowth. We found that GDNF induces neurite outgrowth from hippocampal neurons. The neuritogenic effect of GDNF was independent of Ret, and although the existence of additional GDNF receptors has been indicated (Pozas and Ibáñez, 2005; Cao et al., 2008), we observed complete inhibition of GDNF-induced neurite outgrowth when knocking down NCAM expression, supporting the hypothesis that NCAM mediates GDNF-induced neurite outgrowth in hippocampal neurons, as previously reported by Paratcha et al. (2003). Trans-homophilic NCAM interactions were not required for GDNF-induced neurite outgrowth in neurons grown at low density on plastic or on control fibroblasts. Moreover, we found that GDNF induced additional neurite outgrowth on top of the neurite outgrowth induced by homophilic NCAM interactions. These results indicate that trans-homophilic NCAM interactions do not prevent GDNF from using NCAM as a receptor. Furthermore, we found that removal of PSA from NCAM, which increases homophilic binding, but reduces induction of neurite outgrowth by homophilic interactions (Rutishauser, 2008), does not affect GDNF-induced neurite outgrowth, further indicating that the presence of trans-homophilic NCAM interactions does not prevent GDNF from using NCAM as a receptor. Consistent with these observations, GDNF is reported not to interfere with NCAM-mediated cell adhesion (Paratcha et al., 2003; Ledda et al., 2007).

Previous studies on the interaction between GDNF and NCAM showed that NCAM-140 bound GDNF and mediated the neuritogenic effect of GDNF via activation of Fyn (Paratcha et al., 2003). However, the three major NCAM isoforms have identical ectodomains, and there are no obvious structural reasons why GDNF should not interact with all of them. Here, we uncoupled signaling via NCAM-180 and NCAM-140, respectively, and found that both isoforms are involved in mediating GDNFinduced neurite outgrowth. We did not test the possibility of a direct interaction between GDNF and NCAM-180, but we find that NCAM-180 is involved in the signaling induced by GDNF. Notably, GDNF has been shown to bind to NCAM-120 (Paratcha et al., 2003), and although this interaction might not contribute to GDNF-induced intracellular signaling, it supports the notion that GDNF can bind to other isoforms than NCAM-140. Moreover, stimulation of Schwann cells with GDNF increases the translocation of all major NCAM isoforms into lipid rafts (Iwase et al., 2005), further supporting the hypothesis that GDNFNCAM interactions are not confined to specific NCAM isoforms. In hippocampal neurons, neurite outgrowth induced by ho- mophilic NCAM interactions was recently shown to mainly depend on NCAM-180 (Korshunova et al., 2007). Thus, the involvement of both NCAM-180 and NCAM-140 appears to be a characteristic of GDNF-induced neurite outgrowth, distinguishing it from neurite outgrowth induced by homophilic NCAM interactions.

NCAM polysialylation has profound effects on NCAM function (Rutishauser, 2008) and has been reported to be important for cellular responses to BDNF, CNTF, and PDGF (Vutskits et al., 2001, 2003; Zhang et al., 2004). Similarly, the chemotactic effect of GDNF was found to depend on NCAM polysialylation (Conchonaud et al., 2007). In contrast, we found that GDNF-induced neurite outgrowth occurred independently of NCAM polysialylation. This apparent discrepancy might be explained by the fact that, whereas Conchonaud et al. (2007) studied a directional response, chemotaxis, we investigated an undirectional response, neurite outgrowth, and PSA might not be equally important for both. Supporting this hypothesis, random motility induced by PDGF does not depend on NCAM polysialylation, whereas PDGF-induced chemotaxis does (Zhang et al., 2004).

In addition to NCAM, and possibly GFR $\alpha 1$, other components may be needed for optimal GDNF-induced signaling. We did not investigate the requirements for other receptor components but observed a markedly higher increase in neurite outgrowth from neurons grown on control fibroblasts $(\sim 20 \mu \mathrm{m})$ than from neurons grown on plastic $(\sim 8 \mu \mathrm{m})$. Data from Iwase et al. (2005) suggest that heparan sulfate proteoglycans (HSPGs) are needed for GDNF-induced signaling mediated via NCAM. Fibroblasts produce HSPGs, which may explain why the effect of GDNF is more pronounced when neurons are grown on fibroblasts than when they are grown on plastic. The fibroblasts used in this study also express GFR $\alpha 1$ (supplemental Fig. S1C, available at www.jneurosci.org as supplemental material), and it is possible that binding of GDNF to GFR $\alpha 1$ can locally increase GDNF concentrations, thereby promoting GDNF-induced signaling in the neurons. Alternatively, GDNF bound to GFR $\alpha 1$ might activate NCAM in trans similar to what has been shown for Ret (Paratcha et al., 2001).

Consistent with Paratcha et al. (2003), we found that Fyn is involved in GDNF-induced signaling leading to neurite outgrowth. We also found that FGFR is involved in GDNF-induced NCAM-mediated signaling. Using a pharmacological inhibitor (SU5402) or dnFGFR, we observed an inhibition of GDNFinduced neurite outgrowth. Furthermore, we found that GDNF induced FGFR activation. In contrast, Paratcha et al. (2003) found that SU5402 did not inhibit GDNF-induced neurite outgrowth and accordingly concluded that FGFR was not involved in GDNF-induced neurite outgrowth. We cannot definitely provide an explanation for this difference, with the exception that such a discrepancy might be attributable to differences in the experimental design. We stimulated hippocampal neurons with GDNF in solution, whereas Paratcha et al. grew hippocampal neurons on GDNF-coated plates. Lateral intramembrane mobility is most likely required to bring FGFR receptor monomers into close proximity to achieve receptor activation. This mobility might be different when GDNF is presented as a substrate.

We also investigated the structural basis for the interaction between GDNF and NCAM. Using an SPR-based approach, we found that the NCAM Ig3 module is necessary for GDNF binding, suggesting that motifs within Ig3 are a part of the binding site for GDNF. Whether Ig3 is sufficient for GDNF binding appeared unclear because we did not observe binding of Ig3 itself to GDNF, 
but this could be attributable to limited sensitivity of the applied technique. Notably, soluble Ig3 was able to inhibit GDNFinduced neurite outgrowth, supporting the possibility that Ig3 is sufficient for GDNF binding. Consistent with these findings, Sjöstrand et al. (2007) showed that Ig3 is necessary and sufficient for GDNF binding. Together, these results indicate that the binding site for GDNF is contained within NCAM Ig3.

Based on our SPR data, we found the affinity for GDNF binding to NCAM to be $\sim 1 \mu \mathrm{M}$. In comparison, Paratcha et al. (2003) reported an affinity of $\sim 5 \mathrm{~nm}$ based on the binding of radioactively labeled GDNF to NCAM-expressing cells. The discrepancy is most likely attributable to the fact that we used NCAM fragments (recombinantly produced Ig modules), whereas Paratcha et al. (2003) tested GDNF binding to full-length NCAM expressed on the cell surface of transfected COS cells. Although GDNF has been shown to bind the Ig3 module (Sjöstrand et al., 2007), other NCAM modules in a full-length molecule may modulate the affinity. Additionally, the test systems applied in the two studies have important differences. In SPR analysis, one of the binding partners is immobilized on a sensor chip, and therefore immobile, whereas NCAM molecules expressed on the cell surface have a certain degree of lateral mobility allowing, on binding of GDNF dimers, NCAM clustering, which may increase the measured $K_{\mathrm{D}}$ value.

In contrast to the NCAM binding site for GDNF, the GDNF binding site for NCAM has remained unknown. We ex silico identified a sequence within GDNF that binds to NCAM. The binding of this sequence in peptide form to NCAM resembles that of GDNF, both qualitatively and quantitatively. Furthermore, Gliafin, the peptide form of this sequence, is able to reduce GDNF-induced FGFR phosphorylation, indicating that GDNF and Gliafin compete for the same binding site, further supporting the interpretation that the identified sequence represents a genuine binding site. Molecular modeling has previously been used to identify four residues in GDNF (R39, K81, K125, and $\mathrm{R} 130$ ) that are assumed to be involved in the binding to NCAM (Sjöstrand et al., 2007). K81 is contained within the presently identified sequence and could be involved in NCAM binding. However, a truncated version of Gliafin lacking K81 was as effective as the original Gliafin peptide in inducing neurite outgrowth; therefore, K81 may not be essential for the binding of GDNF to NCAM. In contrast, our data suggest that the residues N85-R88 might be involved in Gliafin binding to NCAM or be important for the secondary structure of the peptide, which we found by circular dichroism spectroscopy to be predominantly $\alpha$-helical.

Our peptides did not contain any of the other three residues identified by molecular modeling. Therefore, we cannot exclude the possibility that these residues may also participate in binding to NCAM. Additional binding sites for NCAM indeed may exist. However, the binding sequence characterized here not only retains the characteristics of GDNF with regard to binding to NCAM, but also mimics GDNF functionally. Thus, we identified a novel GDNF mimetic, termed Gliafin, which will be a useful tool in future studies of GDNF-NCAM interactions and the resulting biological responses.

Notably, based on the structure of the GDNF-GFR $\alpha 1$ complex (Parkash et al., 2008), none of the residues contained in Gliafin are involved in the binding of GDNF to GFR $\alpha 1$ and are thus free to interact with other molecules such as NCAM. GFR $\alpha 1$ have been shown to be necessary for GDNF-induced signaling mediated via NCAM (Paratcha et al., 2003), suggesting that it is most likely also needed for NCAM-mediated neurite outgrowth induced by GDNF. Interestingly, Gliafin, which appears to be separated from the GFR $\alpha 1$ binding part of GDNF, can induce neurite outgrowth via NCAM, suggesting that Gliafin may do so independently of GFR $\alpha 1$. However, this issue should be addressed in future studies.

Our finding that only the oligomeric forms of the Gliafin peptide can induce neurite outgrowth (supplemental Fig. $S 6 D$, available at www.jneurosci.org as supplemental material) suggests that some degree of clustering is required for activation of NCAM by Gliafin or, by inference, GDNF binding. GDNF exists as a homodimer and contains two copies of the presently identified binding site for NCAM. Thus, GDNF can be envisioned to bind to more than one NCAM molecule simultaneously. Because of its modular nature, such NCAM molecules may be concurrently involved in other interactions (e.g., homophilic interactions), and the sum of these interactions could lead to NCAM clustering. Interestingly, these data appear to indicate that, whether NCAM is activated by homophilic binding or by GDNF, clustering of NCAM plays a central role and may provide a mechanism for integrating short- and long-range signals.

\section{References}

Airaksinen MS, Saarma M (2002) The GDNF family: signalling, biological functions and therapeutic value. Nat Rev Neurosci 3:383-394.

Airaksinen MS, Titievsky A, Saarma M (1999) GDNF family neurotrophic factor signaling: four masters, one servant? Mol Cell Neurosci 13:313-325.

Ambjørn M, Asmussen JW, Lindstam M, Gotfryd K, Jacobsen C, Kiselyov VV, Moestrup SK, Penkowa M, Bock E, Berezin V (2008) Metallothionein and a peptide modeled after metallothionein, EmtinB, induce neuronal differentiation and survival through binding to receptors of the low-density lipoprotein receptor family. J Neurochem 104:21-37.

Atkins AR, Osborne MJ, Lashuel HA, Edelman GM, Wright PE, Cunningham BA, Dyson HJ (1999) Association between the first two immunoglobulinlike domains of the neural cell adhesion molecule N-CAM. FEBS Lett 451:162-168.

Atkins AR, Chung J, Deechongkit S, Little EB, Edelman GM, Wright PE, Cunningham BA, Dyson HJ (2001) Solution structure of the third immunoglobulin domain of the neural cell adhesion molecule N-CAM: can solution studies define the mechanism of homophilic binding? J Mol Biol 311:161-172.

Baloh RH, Tansey MG, Johnson EM Jr, Milbrandt J (2000) Functional mapping of receptor specificity domains of glial cell line-derived neurotrophic factor (GDNF) family ligands and production of GFR $\alpha 1$ RET-specific agonists. J Biol Chem 275:3412-3420.

Beggs HE, Soriano P, Maness PF (1994) NCAM-dependent neurite outgrowth is inhibited in neurons from Fyn-minus mice. J Cell Biol 127:825-833.

Beggs HE, Baragona SC, Hemperly JJ, Maness PF (1997) NCAM140 interacts with the focal adhesion kinase p125(fak) and the SRC-related tyrosine kinase p59(fyn). J Biol Chem 272:8310-8319.

Bodrikov V, Leshchyns'ka I, Sytnyk V, Overvoorde J, den Hertog J, Schachner M (2005) RPTPalpha is essential for NCAM-mediated p59fyn activation and neurite elongation. J Cell Biol 168:127-139.

Bonfanti L (2006) PSA-NCAM in mammalian structural plasticity and neurogenesis. Prog Neurobiol 80:129-164.

Büttner B, Horstkorte R (2008) Intracelluar ligands of NCAM. Neurochem Res. Advance online publication. Retrieved September 1, 2009. doi:10.1007/s11064-008-9592-1.

Büttner B, Reutter W, Horstkorte R (2004) Cytoplasmic domain of NCAM 180 reduces NCAM-mediated neurite outgrowth. J Neurosci Res 75:854-860.

Cao JP, Yu JK, Li C, Sun Y, Yuan HH, Wang HJ, Gao DS (2008) Integrin $\beta 1$ is involved in the signaling of glial cell line-derived neurotrophic factor. J Comp Neurol 509:203-210.

Conboy L, Bisaz R, Markram K, Sandi C (2008) Role of NCAM in emotion and learning. Neurochem Res. Advance online publication. Retrieved September 1, 2009. doi:10.1007/s11064-008-9601-4.

Conchonaud F, Nicolas S, Amoureux MC, Ménager C, Marguet D, Lenne PF, Rougon G, Matarazzo V (2007) Polysialylation increases lateral diffu- 
sion of neural cell adhesion molecule in the cell membrane. J Biol Chem 282:26266-26274.

Cunningham BA, Hemperly JJ, Murray BA, Prediger EA, Brackenbury R, Edelman GM (1987) Neural cell adhesion molecule: structure, immunoglobulin-like domains, cell surface modulation, and alternative RNA splicing. Science 236:799-806

Ditlevsen DK, Povlsen GK, Berezin V, Bock E (2008) NCAM-induced intracellular signaling revisited. J Neurosci Res 86:727-743.

Doherty P, Barton CH, Dickson G, Seaton P, Rowett LH, Moore SE, Gower HJ, Walsh FS (1989) Neuronal process outgrowth of human sensory neurons on monolayers of cells transfected with cDNAs for five human N-CAM isoforms. J Cell Biol 109:789-798.

Doherty P, Cohen J, Walsh FS (1990) Neurite outgrowth in response to transfected N-CAM changes during development and is modulated by polysialic acid. Neuron 5:209-219.

Doherty P, Moolenaar CE, Ashton SV, Michalides RJ, Walsh FS (1992a) The VASE exon downregulates the neurite growth-promoting activity of NCAM 140. Nature 356:791-793.

Doherty P, Skaper SD, Moore SE, Leon A, Walsh FS (1992b) A developmentally regulated switch in neuronal responsiveness to NCAM and $\mathrm{N}$-cadherin in the rat hippocampus. Development 115:885-892.

Eketjäll S, Fainzilber M, Murray-Rust J, Ibáñez CF (1999) Distinct structural elements in GDNF mediate binding to GFR $\alpha 1$ and activation of the GFR $\alpha 1-c-R e t$ receptor complex. EMBO J 18:5901-5910.

Enomoto H (2005) Regulation of neural development by glial cell linederived neurotrophic factor family ligands. Anat Sci Int 80:42-52.

Golden JP, DeMaro JA, Osborne PA, Milbrandt J, Johnson EM Jr (1999) Expression of neurturin, GDNF, and GDNF family-receptor mRNA in the developing and mature mouse. Exp Neurol 158:504-528.

Hansen RK, Christensen C, Korshunova I, Kriebel M, Burkarth N, Kiselyov VV, Olsen M, Ostergaard S, Holm A, Volkmer H, Walmod PS, Berezin V, Bock E (2007) Identification of NCAM-binding peptides promoting neurite outgrowth via a heterotrimeric G-protein-coupled pathway. J Neurochem 103:1396-1407.

Hartz BP, Rønn LC (2008) NCAM in long-term potentiation and learning. Neurochem Res. Advance online publication. Retrieved September 1, 2009. doi:10.1007/s11064-008-9820-8

Iwase T, Jung CG, Bae H, Zhang M, Soliven B (2005) Glial cell line-derived neurotrophic factor-induced signaling in Schwann cells. J Neurochem 94:1488-1499.

Jensen PH, Soroka V, Thomsen NK, Ralets I, Berezin V, Bock E, Poulsen FM (1999) Structure and interactions of NCAM modules 1 and 2, basic elements in neural cell adhesion. Nat Struct Biol 6:486-493.

Kasper C, Stahlhut M, Berezin V, Maar TE, Edvardsen K, Kiselyov VV, Soroka V, Bock E (1996) Functional characterization of NCAM fibronectin type III domains: demonstration of modulatory effects of the proline-rich sequence encoded by alternatively spliced exons a and AAG. J Neurosci Res 46:173-186.

Kasper C, Rasmussen H, Kastrup JS, Ikemizu S, Jones EY, Berezin V, Bock E, Larsen IK (2000) Structural basis of cell-cell adhesion by NCAM. Nat Struct Biol 7:389-393.

Kiryushko D, Kofoed T, Skladchikova G, Holm A, Berezin V, Bock E (2003) A synthetic peptide ligand of neural cell adhesion molecule (NCAM), C3d, promotes neuritogenesis and synaptogenesis and modulates presynaptic function in primary cultures of rat hippocampal neurons. J Biol Chem 278:12325-12334.

Kiselyov VV, Berezin V, Maar TE, Soroka V, Edvardsen K, Schousboe A, Bock E (1997) The first immunoglobulin-like neural cell adhesion molecule (NCAM) domain is involved in double-reciprocal interaction with the second immunoglobulin-like NCAM domain and in heparin binding. J Biol Chem 272:10125-10134.

Kiselyov VV, Skladchikova G, Hinsby AM, Jensen PH, Kulahin N, Soroka V, Pedersen N, Tsetlin V, Poulsen FM, Berezin V, Bock E (2003) Structural basis for a direct interaction between FGFR1 and NCAM and evidence for a regulatory role of ATP. Structure 11:691-701.

Kolkova K, Novitskaya V, Pedersen N, Berezin V, BockE (2000a) Neural cell adhesion molecule-stimulated neurite outgrowth depends on activation of protein kinase $\mathrm{C}$ and the Ras-mitogen-activated protein kinase pathway. J Neurosci 20:2238-2246.

Kolkova K, Pedersen N, Berezin V, Bock E (2000b) Identification of an amino acid sequence motif in the cytoplasmic domain of the NCAM-140
$\mathrm{kDa}$ isoform essential for its neuritogenic activity. J Neurochem 75:1274-1282.

Korshunova I, Novitskaya V, Kiryushko D, Pedersen N, Kolkova K, Kropotova E, Mosevitsky M, Rayko M, Morrow JS, Ginzburg I, Berezin V, Bock E (2007) GAP-43 regulates NCAM-180-mediated neurite outgrowth. J Neurochem 100:1599-1612.

Kulahin N, Kasper C, Gajhede M, Berezin V, Bock E, Kastrup JS (2004) Expression, crystallization and preliminary X-ray analysis of extracellular modules of the neural cell-adhesion molecules NCAM and L1. Acta Crystallogr D Biol Crystallogr 60:591-593.

Ledda F, Paratcha G, Sandoval-Guzmán T, Ibáñez CF (2007) GDNF and GFR $\alpha 1$ promote formation of neuronal synapses by ligand-induced cell adhesion. Nat Neurosci 10:293-300.

Liu L, Haines S, Shew R, Akeson RA (1993) Axon growth is enhanced by NCAM lacking the VASE exon when expressed in either the growth substrate or the growing axon. J Neurosci Res 35:327-345.

Maar TE, Rønn LC, Bock E, Berezin V, Moran J, Pasantes-Morales H, Schousboe A (1997) Characterization of microwell cultures of dissociated brain tissue for studies of cell-cell interactions. J Neurosci Res 47:163-172.

Maness PF, Schachner M (2007) Neural recognition molecules of the immunoglobulin superfamily: signaling transducers of axon guidance and neuronal migration. Nat Neurosci 10:19-26.

Mariotti A, Kedeshian PA, Dans M, Curatola AM, Gagnoux-Palacios L, Giancotti FG (2001) EGF-R signaling through Fyn kinase disrupts the function of integrin alpha6beta 4 at hemidesmosomes: role in epithelial cell migration and carcinoma invasion. J Cell Biol 155:447-458.

Neiiendam JL, Køhler LB, Christensen C, Li S, Pedersen MV, Ditlevsen DK, Kornum MK, Kiselyov VV, Berezin V, Bock E (2004) An NCAM-derived FGF-receptor agonist, the FGL-peptide, induces neurite outgrowth and neuronal survival in primary rat neurons. J Neurochem 91:920-935.

Nielsen J, Kulahin N, Walmod PS (2008) Extracellular protein interactions mediated by the neural cell adhesion molecule, NCAM: heterophilic interactions between NCAM and cell adhesion molecules, extracellular matrix proteins, and viruses. Neurochem Res. Advance online publication. Retrieved September 1, 2009. doi:10.1007/s11064-008-9761-2.

Niethammer P, Delling M, Sytnyk V, Dityatev A, Fukami K, Schachner M (2002) Cosignaling of NCAM via lipid rafts and the FGF receptor is required for neuritogenesis. J Cell Biol 157:521-532.

Nosrat CA, Tomac A, Hoffer BJ, Olson L (1997) Cellular and developmental patterns of expression of Ret and glial cell line-derived neurotrophic factor receptor alpha mRNAs. Exp Brain Res 115:410-422.

Paratcha G, Ledda F (2008) GDNF and GFR $\alpha$ : a versatile molecular complex for developing neurons. Trends Neurosci 31:384-391.

Paratcha G, Ledda F, Baars L, Coulpier M, Besset V, Anders J, Scott R, Ibáñez CF (2001) Released GFR $\alpha 1$ potentiates downstream signaling, neuronal survival, and differentiation via a novel mechanism of recruitment of c-Ret to lipid rafts. Neuron 29:171-184.

Paratcha G, Ledda F, Ibáñez CF (2003) The neural cell adhesion molecule NCAM is an alternative signaling receptor for GDNF family ligands. Cell 113:867-879.

Parkash V, Leppanen VM, Virtanen H, Jurvansuu JM, Bespalov MM, Sidorova YA, Runeberg-Roos P, Saarma M, Goldman A (2008) The structure of the GDNF:coreceptor complex: insights into RET signalling and heparin binding. J Biol Chem 283:35164-35172.

Pezeshki G, Franke B, Engele J (2001) Evidence for a ligand-specific signaling through GFR $\alpha-1$, but not GFR $\alpha-2$, in the absence of Ret. J Neurosci Res 66:390-395

Poteryaev D, Titievsky A, Sun YF, Thomas-Crusells J, Lindahl M, Billaud M, Arumäe U, Saarma M (1999) GDNF triggers a novel ret-independent Src kinase family-coupled signaling via a GPI-linked GDNF receptor $\alpha 1$. FEBS Lett 463:63-66.

Pozas E, Ibáñez CF (2005) GDNF and GFR $\alpha 1$ promote differentiation and tangential migration of cortical GABAergic neurons. Neuron 45:701-713.

Prag S, Lepekhin EA, Kolkova K, Hartmann-Petersen R, Kawa A, Walmod PS Belman V, Gallagher HC, Berezin V, Bock E, Pedersen N (2002) NCAM regulates cell motility. J Cell Sci 115:283-292.

Rønn LC, Ralets I, Hartz BP, Bech M, Berezin A, Berezin V, Møller A, Bock E (2000) A simple procedure for quantification of neurite outgrowth based on stereological principles. J Neurosci Methods 100:25-32.

Rutishauser U (2008) Polysialic acid in the plasticity of the developing and adult vertebrate nervous system. Nat Rev Neurosci 9:26-35.

Saffell JL, Walsh FS, Doherty P (1994) Expression of NCAM containing VASE 
in neurons can account for a developmental loss in their neurite outgrowth response to NCAM in a cellular substratum. J Cell Biol 125:427-436.

Saffell JL, Williams EJ, Mason IJ, Walsh FS, Doherty P (1997) Expression of a dominant negative FGF receptor inhibits axonal growth and FGF receptor phosphorylation stimulated by CAMs. Neuron 18:231-242.

Sjöstrand D, Carlsson J, Paratcha G, Persson B, Ibáñez CF (2007) Disruption of the GDNF binding site in NCAM dissociates ligand binding and homophilic cell adhesion. J Biol Chem 282:12734-12740.

Soroka V, Kolkova K, Kastrup JS, Diederichs K, Breed J, Kiselyov VV, Poulsen FM, Larsen IK, Welte W, Berezin V, Bock E, Kasper C (2003) Structure and interactions of NCAM Ig1-2-3 suggest a novel zipper mechanism for homophilic adhesion. Structure 11:1291-1301.

Soroka V, Kasper C, Poulsen FM (2008) Structural biology of NCAM. Neurochem Res. Advance online publication. Retrieved September 1, 2009. doi:10.1007/s11064-008-9837-z.

Trupp M, Belluardo N, Funakoshi H, Ibáñez CF (1997) Complementary and overlapping expression of glial cell line-derived neurotrophic factor (GDNF), c-ret proto-oncogene, and GDNF receptor- $\alpha$ indicates multiple mechanisms of trophic actions in the adult rat CNS. J Neurosci 17:3554-3567.
Trupp M, Scott R, Whittemore SR, Ibáñez CF (1999) Ret-dependent and -independent mechanisms of glial cell line-derived neurotrophic factor signaling in neuronal cells. J Biol Chem 274:20885-20894.

Vutskits L, Djebbara-Hannas Z, Zhang H, Paccaud JP, Durbec P, Rougon G, Muller D, Kiss JZ (2001) PSA-NCAM modulates BDNF-dependent survival and differentiation of cortical neurons. Eur J Neurosci 13:1391-1402.

Vutskits L, Gascon E, Kiss JZ (2003) Removal of PSA from NCAM affects the survival of magnocellular vasopressin- and oxytocin-producing neurons in organotypic cultures of the paraventricular nucleus. Eur J Neurosci 17:2119-2126.

Walmod PS, Kolkova K, Berezin V, Bock E (2004) Zippers make signals: NCAM-mediated molecular interactions and signal transduction. Neurochem Res 29:2015-2035.

Yu T, Scully S, Yu Y, Fox GM, Jing S, Zhou R (1998) Expression of GDNF family receptor components during development: implications in the mechanisms of interaction. J Neurosci 18:4684-4696.

Zhang H, Vutskits L, Calaora V, Durbec P, Kiss JZ (2004) A role for the polysialic acid-neural cell adhesion molecule in PDGF-induced chemotaxis of oligodendrocyte precursor cells. J Cell Sci 117:93-103. 